\title{
Onset of thermal convection in a rectangular parallelepiped cavity of small aspect ratios
}

\author{
AUTHOR(S): \\ Funakoshi, Mitsuaki
}

CITATION:

Funakoshi, Mitsuaki. Onset of thermal convection in a rectangular parallelepiped cavity of small aspect ratios. Fluid Dynamics Research 2018, 50(2): 021402.

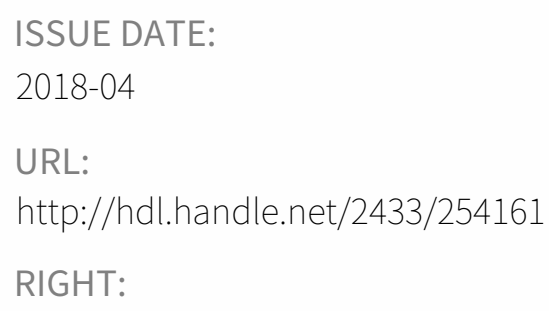

This is an author-created, un-copyedited version of an article accepted for publication in 'Fluid Dynamics Research'. The publisher is not responsible for any errors or omissions in this version of the manuscript or any version derived from it. The Version of Record is available online at https://doi.org/10.1088/1873-7005/aaa194;; The full-text file will be made open to the public on 6 February 2019 in accordance with publisher's 'Terms and Conditions for Self-Archiving'.; This is not the published version. Please cite only the published version.; この論文は出版社版でありません。引用の際には出 版社版をご確認ご利用ください。 


\title{
Onset of Thermal Convection in a Rectangular Parallelepiped Cavity of Small Aspect Ratios
}

\author{
Mitsuaki Funakoshi \\ Professor Emeritus, Kyoto University, Yoshida-Honmachi, Sakyo-ku, Kyoto 606-8501, \\ Japan \\ E-mail: funakoshi.mitsuaki.56m@st.kyoto-u.ac.jp
}

\begin{abstract}
Onset of thermal convection of a fluid in a rectangular parallelepiped cavity of small aspect ratios is examined both numerically and analytically under the assumption that its all walls are rigid and of perfect thermal conductance exposed to a vertically linear temperature field. Critical Rayleigh number $R_{c}$ and the steady velocity and temperature fields of most unstable modes are computed by a Galerkin spectral method of high accuracy for aspect ratios $A_{x}$ and $A_{y}$ either or both of which are small.

We find that if $A_{x}$ is decreased to 0 with $A_{y}$ being kept constant, $R_{c}$ increases proportionally to $A_{x}^{-4}$, the convection rolls of most unstable mode whose axes are parallel to the shorter side walls become narrower, and their number increases proportionally to $A_{x}^{-\frac{1}{2}}$. Moreover, as $A_{x}$ is decreased, we observe the changes of the symmetry of most unstable mode that occur more frequently for smaller $A_{x}$. However, if $A_{x}=A_{y}=A$ is decreased to 0 , although we again observe the increase in $R_{c}$ proportional to $A^{-4}$, we obtain only one narrow convection roll as the velocity field of most unstable mode for all $A$.

The expressions of $R_{c}$ and velocity fields in the limit of $A_{x} \rightarrow 0$ or $A \rightarrow 0$ are obtained by an asymptotic analysis in which the dependences of $R_{c}$ and the magnitude and length scale of velocity fields of most unstable modes on $A_{x}$ and $A_{y}$ in the numerical computations are used. For example, $R_{c}$ is approximated by $\pi^{4} A_{x}^{-4}$ and $25 \pi^{4} A^{-4}$ in the limits of $A_{x} \rightarrow 0$ and $A \rightarrow 0$, respectively. Moreover, analytical expressions of some components of velocity fields in these limits are derived. Finally, we find that for small $A_{x}$ or $A$ the agreement between the numerical and analytical results on $R_{c}$ and velocity field is quite good except for the velocity field in thin wall layers near the top and bottom walls.
\end{abstract}




\section{Introduction}

Because the onset of thermal convection of a fluid in a closed cavity is an interesting problem in fluid dynamics and also important in applications, it has been investigated theoretically, numerically or experimentally in several studies for many years (see, for example, Gershuni and Zhukhovitskii (1972), Koschmieder (1993), Lappa (2010), and references therein.). Some of these studies examined the onset of three-dimensional thermal convection in a rectangular parallelepiped cavity of rigid walls for several values of aspect ratios $A_{x}=L_{x} / d$ and $A_{y}=L_{y} / d$, where $L_{x}$ and $L_{y}$ are horizontal lengths of the cavity, and $d$ is its height. Koschmieder (1966) experimentally investigated this problem and showed that if both $A_{x}$ and $A_{y}$ are larger than 1 and the difference between them is not small, the pattern of flows that appears first as the difference in temperatures on upper and lower walls is increased slowly is a linear arrangement of several convection rolls of nearly square cross-section whose axes are horizontal and parallel to shorter side walls. Stork and Müller (1972) also obtained similar results for $A_{x}$ and $A_{y}$ larger than 1 in their experiments on the onset of three-dimensional thermal convection in a rectangular parallelepiped cavity with side walls where an essentially linear vertical temperature profile is maintained. Moreover, they pointed out that if one of the aspect ratios is smaller than 1, the widths of observed convection rolls whose axes are parallel to the shorter side walls are smaller than their height. For example, in the experiments with $A_{y}$ between 3 and 6 , they found that the number of observed convection rolls is larger than $A_{y}$ if $A_{x}$ is 0.7 or 0.5 , although detailed examination of convection rolls for $A_{x}$ less than 0.5 was not performed. They also observed the tendency that $R_{c}$ increases rapidly as $A_{x}$ decreases from 1 with $A_{y}$ being kept constant.

Davis (1967) numerically examined the onset of thermal convection in a rectangular parallelepiped cavity with walls of perfect thermal conductance. If the temperatures on upper and lower walls are constants, and the temperature on side walls is a linear function of vertical coordinate connecting these temperatures, the motionless state (thermal conduction state) of a fluid in the cavity is possible. He examined the critical Rayleigh number $R_{c}$ of this state and the flow pattern of most unstable mode that is destabilized at this Rayleigh number for several values of aspect ratios $A_{x}$ and $A_{y}$. In this examination, he adopted the assumption of finite roll, in which a horizontal component of fluid velocity normal to a side wall is 0 , and obtained the results that $R_{c}$ rapidly increases with the decrease in one of $A_{x}$ and $A_{y}$ and that the change of most unstable modes frequently occurs with their decrease to 0 . Moreover, he found that if both $A_{x}$ and $A_{y}$ are larger than 1 and the difference between them is not small, the flow patterns of most unstable mode are similar to those observed in the experiments mentioned above at the onset of thermal convection. When either $A_{x}$ or $A_{y}$ is smaller than 1 , he obtained the flow patterns of most unstable mode composed of a few narrow convection rolls for which their height is larger than their width, which is consistent with the experimental observation by Stork and Müller (1972). Although Davis (1967) obtained the above remarkable and interesting results, his study is not perfect because 
the assumption of finite roll cannot be satisfied exactly by the solutions to governing equations of thermal convection, as was pointed out by Davies-Jones (1970).

After the study by Davis (1967), there were several numerical studies aiming at the determination of $R_{c}$ and/or most unstable modes for many values of aspect ratios of a three-dimensional rectangular or cubic cavity with rigid walls of perfect thermal conductance (see, for example, Catton (1970), Gershuni and Zhukhovitskii (1972) and Kirchartz and Oertel (1988)). The computation of $R_{c}$ and most unstable modes with high accuracy was carried out by Mizushima and Matsuda $(1996,1997)$ for cubic and rectangular parallelepiped cavities. Fukazawa and Funakoshi (2015) systematically examined $R_{c}$ and the flow patterns of most unstable mode for several values of $A_{x}$ and $A_{y}$ using a Galerkin spectral method of high accuracy. They found the tendency that $R_{c}$ increases rapidly as either $A_{x}$ or $A_{y}$ decreases to 0 , and decreases slowly as both $A_{x}$ and $A_{y}$ increase. They examined the flow pattern of most unstable mode in detail for $1 \leq A_{x}, A_{y} \leq 6$, and showed that the flow pattern is a linear arrangement of several convection rolls of nearly square cross-section whose axes are horizontal and parallel to shorter side walls if the difference between $A_{x}$ and $A_{y}$ is not small. They also observed frequent change of most unstable mode and rapid increase in $R_{c}$ with the decrease in either $A_{x}$ or $A_{y}$ from 1.

The onset of thermal convection in a three-dimensional rectangular or cubic cavity for different boundary conditions was also examined numerically in several studies. For example, Edwards (1988) obtained $R_{c}$ and the flow patterns of most unstable mode for $A_{x}$ and $A_{y}$ between 1 and 12 under the assumption of adiabatic side walls. Mizushima and Nakamura (2003) also examined the dependence of $R_{c}$ and the flow patterns on $A_{x}$ and $A_{y}$ that are 1 or more for the same thermal boundary condition on side walls. The Bénard-Marangoni instability in a rectangular parallelepiped cavity for $A_{x}$ and $A_{y}$ between 1 and 9 was examined by Dauby and Lebon (1996) for adiabatic side walls and a convective cooling condition on the top wall. Gelfgat (1999) examined the $R_{c}$ and symmetries of most unstable modes for $A_{x}$ and $A_{y}$ larger than 1 for adiabatic side walls and stress-free and convective cooling conditions on the top wall. Moreover, there are several numerical studies on the bifurcations of steady solutions of nonlinear governing equations of thermal convection in three-dimensional cubic or rectangular cavities for different boundary conditions on the walls (for example, see Puigjaner et al. (2006, 2008), Lappa (2010), and references therein). However, the case of small aspect ratios was not examined in the above linear stability analysis and bifurcation analysis.

There are also numerical and analytical studies on $R_{c}$ and the flow pattern of most unstable mode of thermal convection in an infinite channel of rectangular crosssection. Chana and Daniels (1989) examined this problem for rigid or stress-free boundary condition on the top and bottom walls under the assumptions of rigid side walls, no volume flux down the channel, and perfect thermal conductance on all the walls. For rigid top and bottom walls, they numerically calculated $R_{c}$ and critical wavenumber $k_{c}$ (non-dimensionalized with $d$ ) for several values of aspect ratio $A_{x}=L_{x} / d$ of cross-section, where $L_{x}$ and $d$ are horizontal and vertical length of the 
cross-section, respectively. They found that $R_{c}$ rapidly increases as $A_{x}$ decreases from 1 and that for sufficiently small $A_{x}, k_{c}$ increases as $A_{x}$ decreases. They also examined the characteristics of velocity and temperature fields of most unstable mode mainly for $A_{x} \geq 1$. Moreover, they performed an asymptotic analysis in which the asymptotic forms of $R_{c}, k_{c}$, and velocity and temperature fields of most unstable mode in the limits of $A_{x} \rightarrow 0$ and $A_{x} \rightarrow \infty$ were obtained. Daniels and Ong (1990) also numerically computed the values of $R_{c}$ and $k_{c}$ with higher accuracy than Chana and Daniels (1989) for a few $A_{x}$ 's between $1 / 2$ and 4 , and compared these values with the above asymptotic forms of $R_{c}$ and $k_{c}$.

In the preceding numerical studies introduced above, the dependence of $R_{c}$ and the velocity field of most unstable mode on aspect ratios $A_{x}$ and $A_{y}$ of a rectangular parallelepiped cavity was not examined in detail when either or both of $A_{x}$ and $A_{y}$ are smaller than 1. However, the rapid increase in $R_{c}$ and the increase in the number of convection rolls of most unstable mode and their narrowing with the decrease in either $A_{x}$ or $A_{y}$ from 1 are interesting behavior. Therefore, in the present study, we first aim at making this behavior clearer by the detailed examination of $R_{c}$ and the velocity field of most unstable mode for such $A_{x}$ and $A_{y}$ using a numerical analysis of high accuracy and an asymptotic analysis. Moreover, since the rapid increase in $R_{c}$ with the decrease in $A_{x}=A_{y}=A$ from 1 and the formation of one tall vortex roll for small $A$ are also interesting, we also aim at describing these behaviors quantitatively by using the same numerical and analytical methods.

After the description of formulation of the considered problem in section 2, section 3 is devoted to the introduction of the numerical method used to examine the linear stability of the motionless state. The convergence of numerical solutions is shown in section 4. Numerical results on $R_{c}$ and the velocity field of most unstable mode are shown in section 5. In section 6 , using the dependences of $R_{c}$ and the velocity and temperature fields of most unstable modes on $A_{x}$ and $A_{y}$ in the numerical computations, the asymptotic forms of these fields and $R_{c}$ in the limits of $A_{x} \rightarrow 0$ and $A \rightarrow 0$ are obtained by an asymptotic analysis, and are compared with the numerical results shown in section 5. Finally, section 7 is devoted to discussion and conclusions.

\section{Formulation}

We consider the onset of thermal convection of an incompressible viscous fluid in a rectangular parallelepiped cavity exposed to a vertically linear temperature field. Cartesian coordinates $\left(x^{*}, y^{*}, z^{*}\right)$ are defined in which $x^{*}$ and $y^{*}$ axes are horizontal and parallel to the side walls of the cavity, $z^{*}$ axis is vertically upward, and the origin is at the center of the cavity. Two aspect ratios of the cavity are defined as $A_{x} \equiv L_{x} / d$ and $A_{y} \equiv L_{y} / d$, where $L_{x}, L_{y}$ and $d$ respectively denote the length of the cavity in the $x^{*}, y^{*}$ and $z^{*}$ directions. We assume that external temperature field $T_{\mathrm{ex}}^{*}$ is expressed as

$$
T_{\mathrm{ex}}^{*}\left(z^{*}\right)=-\beta z^{*}+T_{0}^{*}
$$


where $\beta(>0)$ is the gradient of the external temperature, and $T_{0}^{*}$ is the external temperature at $z^{*}=0$. All the walls of cavity are assumed to be rigid and of perfect thermal conductance.

Under the Boussinesq approximation, non-dimensionalized forms of the equation of continuity, Navier-Stokes equation and energy equation are written as

$$
\begin{aligned}
& \nabla \cdot \boldsymbol{v}=0 \\
& \frac{1}{P}\left(\frac{\partial}{\partial t}+\boldsymbol{v} \cdot \nabla\right) \boldsymbol{v}=-\nabla p+\Delta \boldsymbol{v}-\left\{\frac{g d^{3}}{\kappa \nu}-R\left(T-T_{0}\right)\right\} \boldsymbol{e}_{z}, \\
& \left(\frac{\partial}{\partial t}+\boldsymbol{v} \cdot \nabla\right) T=\Delta T
\end{aligned}
$$

where

$$
\nabla \equiv \boldsymbol{e}_{x} \frac{\partial}{\partial x}+\boldsymbol{e}_{y} \frac{\partial}{\partial y}+\boldsymbol{e}_{z} \frac{\partial}{\partial z}, \quad \Delta \equiv \frac{\partial^{2}}{\partial x^{2}}+\frac{\partial^{2}}{\partial y^{2}}+\frac{\partial^{2}}{\partial z^{2}},
$$

and velocity $\boldsymbol{v}^{*}$, temperature $T^{*}$, pressure $p^{*}$ of fluid, spatial coordinates $\left(x^{*}, y^{*}, z^{*}\right)$, and the time $t^{*}$ are non-dimensionalized as

$$
x \equiv \frac{x^{*}}{d}, y \equiv \frac{y^{*}}{d}, z \equiv \frac{z^{*}}{d}, t \equiv \frac{\kappa}{d^{2}} t^{*}, \boldsymbol{v} \equiv \frac{d}{\kappa} \boldsymbol{v}^{*}, T \equiv \frac{T^{*}}{\beta d}, p \equiv \frac{d^{2}}{\rho_{0} \kappa \nu} p^{*} .
$$

Moreover, $\boldsymbol{e}_{x}, \boldsymbol{e}_{y}$ and $\boldsymbol{e}_{z}$ respectively represent the unit vectors in the $x^{*}, y^{*}$ and $z^{*}$ directions, and $\rho_{0}$ is the density of fluid for $T^{*}=T_{0}^{*}$. Also $\nu, \alpha$ and $\kappa$ respectively denote the kinematic viscosity, the coefficient of thermal expansion, and the thermal diffusivity of fluid, $g$ is the acceleration due to gravity, and $T_{0} \equiv T_{0}^{*} /(\beta d)$. Two non-dimensional parameters $R$ and $P$ in equation (3) respectively denote the Rayleigh number and the Prandtl number defined by

$$
R \equiv \frac{\alpha g \beta d^{4}}{\kappa \nu}, \quad P \equiv \frac{\nu}{\kappa} .
$$

Non-dimensionalized boundary conditions for the velocity and temperature on the walls of the cavity are expressed as

$$
\boldsymbol{v}=0, T=T_{\mathrm{ex}}(z) \quad \text { on } x= \pm \frac{A_{x}}{2}, \text { on } y= \pm \frac{A_{y}}{2}, \text { and on } z= \pm \frac{1}{2},
$$

where

$$
T_{\mathrm{ex}}(z)=-z+T_{0}
$$

If we define the deviations of velocity, temperature and pressure from those in the motionless state as

$$
\boldsymbol{u} \equiv \boldsymbol{v}-\boldsymbol{v}_{s}, \quad \theta \equiv T-T_{s}, \quad q \equiv p-p_{s},
$$

we obtain the following governing equations:

$$
\begin{aligned}
& \nabla \cdot \boldsymbol{u}=0, \\
& \frac{1}{P}\left(\frac{\partial}{\partial t}+\boldsymbol{u} \cdot \nabla\right) \boldsymbol{u}=-\nabla q+\Delta \boldsymbol{u}+R \theta \boldsymbol{e}_{z},
\end{aligned}
$$




$$
\left(\frac{\partial}{\partial t}+\boldsymbol{u} \cdot \nabla\right) \theta=\Delta \theta+\boldsymbol{u} \cdot \boldsymbol{e}_{z}
$$

where

$$
\boldsymbol{v}_{s}=0, T_{s}=T_{\mathrm{ex}}(z), p_{s}=-\frac{1}{2} R z^{2}-\frac{g d^{3}}{\kappa \nu} z+p_{0},
$$

and $p_{0}$ is the pressure at $z=0$. Boundary conditions for $\boldsymbol{u}$ and $\theta$ are written as

$$
\boldsymbol{u}=0, \theta=0 \quad \text { on } x= \pm \frac{A_{x}}{2}, \text { on } y= \pm \frac{A_{y}}{2}, \text { and on } z= \pm \frac{1}{2},
$$

\section{Stability analysis of motionless state}

We examine the linear stability of the motionless state on the basis of linearized version of equations (9)-(11) and boundary condition (13). The variables $\boldsymbol{u}, \theta$ and $q$ in these equations are assumed to be of the following time dependences:

$$
\boldsymbol{u}=e^{\lambda t} \hat{\boldsymbol{u}}+\text { c.c., } \quad \theta=e^{\lambda t} \hat{\theta}+\text { c.c. }, \quad q=e^{\lambda t} \hat{q}+\text { c.c. },
$$

where $\hat{\boldsymbol{u}}, \hat{\theta}$ and $\hat{q}$ are complex-valued functions of $x, y$ and $z$. Also $\lambda$ is a complex constant, and c.c. denotes the complex conjugate of preceding terms. Critical Rayleigh number $R_{c}$ is defined as the value of $R$ for which the motionless state is neutrally stable, that is, the real part of $\lambda$ equals to 0 . Sherman and Ostrach (1966) showed that $\lambda$ is always real for positive $R$ if the linearized version of equations (9)-(11) and boundary condition (13) are satisfied. Therefore, the value of $R_{c}$ is obtained under the condition of $\lambda=0$.

Governing equations for $\hat{\boldsymbol{u}}, \hat{\theta}$ and $\hat{q}$ of neutrally stable disturbances are expressed as

$$
\begin{aligned}
& \nabla \cdot \hat{\boldsymbol{u}}=0, \\
& -\nabla \hat{q}+\Delta \hat{\boldsymbol{u}}+R_{c} \hat{\theta} \boldsymbol{e}_{z}=0, \\
& \Delta \hat{\theta}+\hat{\boldsymbol{u}} \cdot \boldsymbol{e}_{z}=0
\end{aligned}
$$

Here $\hat{\boldsymbol{u}}, \hat{\theta}$ and $\hat{q}$ can be assumed to be real functions. The boundary conditions for $\hat{\boldsymbol{u}}$ and $\hat{\theta}$ are written as

$$
\hat{\boldsymbol{u}}=0, \hat{\theta}=0 \quad \text { on } x= \pm \frac{A_{x}}{2}, \text { on } y= \pm \frac{A_{y}}{2}, \text { and on } z= \pm \frac{1}{2} .
$$

We use a Galerkin spectral method to solve numerically equations (15)-(17) under boundary condition (18). Since this method is the same as the one used in Fukazawa and Funakoshi (2015), only its main part is described here. We expand $\hat{\boldsymbol{u}}$ and $\hat{\theta}$ as

$$
\begin{aligned}
& \hat{\boldsymbol{u}}(x, y, z)=\sum_{\ell=0}^{2 L+1} \sum_{m=0}^{2 M+1} \sum_{n=0}^{2 N+1} \sum_{J=1}^{3} a_{(\ell, m, n)}^{(J)} \boldsymbol{f}_{(\ell, m, n)}^{(J)}(x, y, z), \\
& \hat{\theta}(x, y, z)=\sum_{\ell=0}^{2 L+1} \sum_{m=0}^{2 M+1} \sum_{n=0}^{2 N+1} b_{(\ell, m, n)} f_{(\ell, m, n)}(x, y, z),
\end{aligned}
$$


where basis functions $\boldsymbol{f}_{(\ell, m, n)}^{(J)}(x, y, z)$ and $f_{(\ell, m, n)}(x, y, z)$ are defined by

$$
\left\{\begin{array}{l}
\boldsymbol{f}_{(\ell, m, n)}^{(1)}(x, y, z) \equiv \nabla \times F_{\ell}\left(\frac{2}{A_{x}} x\right) G_{m}\left(\frac{2}{A_{y}} y\right) G_{n}(2 z) \boldsymbol{e}_{x} \\
\boldsymbol{f}_{(\ell, m, n)}^{(2)}(x, y, z) \equiv \nabla \times G_{\ell}\left(\frac{2}{A_{x}} x\right) F_{m}\left(\frac{2}{A_{y}} y\right) G_{n}(2 z) \boldsymbol{e}_{y} \\
\boldsymbol{f}_{(\ell, m, n)}^{(3)}(x, y, z) \equiv \nabla \times G_{\ell}\left(\frac{2}{A_{x}} x\right) G_{m}\left(\frac{2}{A_{y}} y\right) F_{n}(2 z) \boldsymbol{e}_{z} \\
f_{(\ell, m, n)}(x, y, z) \equiv F_{\ell}\left(\frac{2}{A_{x}} x\right) F_{m}\left(\frac{2}{A_{y}} y\right) F_{n}(2 z) .
\end{array}\right.
$$

Therefore, equation (15) is satisfied for any values of expansion coefficients $a_{(\ell, m, n)}^{(J)}$. In the expression of $\hat{\boldsymbol{u}}$ given by equations (19) and (21), we use three (rather than two) vector potentials so that we can impose decoupled boundary conditions for them on all the walls of the cavity without a loss of generality. In the above basis functions, modified Chebyshev polynomials $F_{\ell}(\xi)$ and $G_{\ell}(\xi)$ defined by

$$
F_{\ell}(\xi) \equiv\left(1-\xi^{2}\right) T_{\ell}(\xi), \quad G_{\ell}(\xi) \equiv\left(1-\xi^{2}\right)^{2} T_{\ell}(\xi), \quad(\ell=0,1,2, \ldots),
$$

are used, where $T_{\ell}(\xi)$ is the Chebyshev polynomial of the $\ell$-th order, and $\xi=$ $2 x / A_{x}, 2 y / A_{y}$ or $2 z$. Therefore, boundary condition (18) is satisfied for any values of expansion coefficients $a_{(\ell, m, n)}^{(J)}$ and $b_{(\ell, m, n)}$. Expansions of $\hat{\boldsymbol{u}}$ and $\hat{\theta}$ are truncated at $\ell=2 L+1, m=2 M+1$ and $n=2 N+1$ in the $x, y$ and $z$ directions, respectively.

Here, we introduce functions $\tilde{\boldsymbol{f}}_{(i, j, k)}^{(I)}(I=1,2,3)$ and $\tilde{f}_{(i, j, k)}$ for $i=0,1, \ldots, 2 L+$ $1, j=0,1, \ldots, 2 M+1$ and $k=0,1, \ldots, 2 N+1$ defined by

$$
\begin{aligned}
& \tilde{\boldsymbol{f}}_{(i, j, k)}^{(I)}(x, y, z) \equiv \begin{cases}\nabla \times \tilde{F}_{i}\left(\frac{2}{A_{x}} x\right) \tilde{G}_{j}\left(\frac{2}{A_{y}} y\right) \tilde{G}_{k}(2 z) \boldsymbol{e}_{x} & (\text { for } I=1), \\
\nabla \times \tilde{G}_{i}\left(\frac{2}{A_{x}} x\right) \tilde{F}_{j}\left(\frac{2}{A_{y}} y\right) \tilde{G}_{k}(2 z) \boldsymbol{e}_{y} & (\text { for } I=2), \\
\nabla \times \tilde{G}_{i}\left(\frac{2}{A_{x}} x\right) \tilde{G}_{j}\left(\frac{2}{A_{y}} y\right) \tilde{F}_{k}(2 z) \boldsymbol{e}_{z} & (\text { for } I=3),\end{cases} \\
& \tilde{f}_{(i, j, k)}(x, y, z) \equiv \tilde{F}_{i}\left(\frac{2}{A_{x}} x\right) \tilde{F}_{j}\left(\frac{2}{A_{y}} y\right) \tilde{F}_{k}(2 z),
\end{aligned}
$$

where functions $\tilde{F}_{i}(\xi)$ and $\tilde{G}_{i}(\xi)$ are defined as

$$
\tilde{F}_{i}(\xi) \equiv \frac{F_{i}(\xi)}{\sqrt{1-\xi^{2}}}, \quad \tilde{G}_{i}(\xi) \equiv \frac{G_{i}(\xi)}{\sqrt{1-\xi^{2}}}, \quad(i=0,1,2, \ldots),
$$

for $\xi=2 x / A_{x}, 2 y / A_{y}$ or $2 z$. To obtain linear algebraic equations for expansion coefficients $a_{(\ell, m, n)}^{(J)}$ and $b_{(\ell, m, n)}$, we impose the following conditions:

$$
\begin{array}{r}
\left\langle\left\langle\tilde{\boldsymbol{f}}_{(i, j, k)}^{(I)},-\nabla \hat{q}+\Delta \hat{\boldsymbol{u}}+R_{c} \hat{\theta} \boldsymbol{e}_{z}\right\rangle\right\rangle=0 \quad(\text { for } \quad I=1,2,3, i=0,1, \ldots, 2 L+1, \\
j=0,1, \ldots, 2 M+1 \text { and } k=0,1, \ldots, 2 N+1), \\
\left\langle\tilde{f}_{(i, j, k)}, \Delta \hat{\theta}+\hat{\boldsymbol{u}} \cdot \boldsymbol{e}_{z}\right\rangle=0 \quad(\text { for } \quad i=0,1, \ldots, 2 L+1, \\
\quad j=0,1, \ldots, 2 M+1 \text { and } k=0,1, \ldots, 2 N+1),
\end{array}
$$


where the inner product $\langle\langle\boldsymbol{f}, \boldsymbol{g}\rangle\rangle$ of vector-valued functions $\boldsymbol{f}(x, y, z)$ and $\boldsymbol{g}(x, y, z)$ is defined by

$$
\langle\langle\boldsymbol{f}, \boldsymbol{g}\rangle\rangle \equiv \int_{-\frac{A_{x}}{2}}^{\frac{A_{x}}{2}} \mathrm{~d} x \int_{-\frac{A_{y}}{2}}^{\frac{A_{y}}{2}} \mathrm{~d} y \int_{-\frac{1}{2}}^{\frac{1}{2}} \mathrm{~d} z \boldsymbol{f}(x, y, z) \cdot \boldsymbol{g}(x, y, z),
$$

and the inner product $\langle f, g\rangle$ of scalar functions $f(x, y, z)$ and $g(x, y, z)$ is defined by

$$
\langle f, g\rangle \equiv \int_{-\frac{A_{x}}{2}}^{\frac{A_{x}}{2}} \mathrm{~d} x \int_{-\frac{A_{y}}{2}}^{\frac{A_{y}}{2}} \mathrm{~d} y \int_{-\frac{1}{2}}^{\frac{1}{2}} \mathrm{~d} z f(x, y, z) g(x, y, z) .
$$

The inner product of $\tilde{\boldsymbol{f}}_{(i, j, k)}^{(I)}$ and the term of pressure gradient in equation (24) vanishes for all $I, i, j$ and $k$, because $\tilde{\boldsymbol{f}}_{(i, j, k)}^{(I)}$ satisfies conditions $\tilde{\boldsymbol{f}}_{(i, j, k)}^{(I)}=0$ (on the walls) and $\nabla \cdot \tilde{\boldsymbol{f}}_{(i, j, k)}^{(I)}=0$ (in the cavity).

Equations (24) and (25) are reduced to a generalized eigenvalue problem expressed as

$$
A \boldsymbol{x}=\sqrt{R_{c}} B \boldsymbol{x}
$$

where eigenvector $\boldsymbol{x}$ has $N_{t} \equiv 4(2 L+2)(2 M+2)(2 N+2)$ components, and $A$ and $B$ are square matrices of dimension $N_{t}$. The $(J-1)(2 L+2)(2 M+2)(2 N+2)+\ell(2 M+$ $2)(2 N+2)+m(2 N+2)+n+1$-th component of eigenvector $\boldsymbol{x}$ expressed as $x_{(J, \ell, m, n)}$ is given by

$$
x_{(J, \ell, m, n)}=\left\{\begin{array}{l}
a_{(\ell, m, n)}^{(J)} \quad(J=1,2,3), \\
\sqrt{R_{c}} b_{(\ell, m, n)} \quad(J=4),
\end{array}\right.
$$

for $\ell=0,1, \ldots, 2 L+1, m=0,1, \ldots, 2 M+1$ and $n=0,1, \ldots, 2 N+1$. The components of matrices $A$ and $B$ in the $(I-1)(2 L+2)(2 M+2)(2 N+2)+i(2 M+2)(2 N+2)+j(2 N+2)+$ $k+1$-th row and $(J-1)(2 L+2)(2 M+2)(2 N+2)+\ell(2 M+2)(2 N+2)+m(2 N+2)+n+1$ -th column, expressed as $a_{(I, i, j, k),(J, \ell, m, n)}$ and $b_{(I, i, j, k),(J, \ell, m, n)}$ for $A$ and $B$ respectively, are written as

$$
\begin{aligned}
& a_{(I, i, j, k),(J, \ell, m, n)}=\left\{\begin{array}{l}
\left\langle\left\langle\tilde{\boldsymbol{f}}_{(i, j, k)}^{(I)}, \Delta \boldsymbol{f}_{(\ell, m, n)}^{(J)}\right\rangle\right\rangle \quad(\text { for } I=1,2,3, J=1,2,3), \\
\left\langle\tilde{f}_{(i, j, k)}, \Delta f_{(\ell, m, n)}\right\rangle \quad(\text { for } I=J=4), \\
0 \quad \text { (otherwise), }
\end{array}\right. \\
& b_{(I, i, j, k),(J, \ell, m, n)}=\left\{\begin{array}{l}
-\left\langle\left\langle\tilde{\boldsymbol{f}}_{(i, j, k)}^{(I)}, f_{(\ell, m, n)} \boldsymbol{e}_{z}\right\rangle\right\rangle \quad(\text { for } I=1,2,3, J=4), \\
-\left\langle\tilde{f}_{(i, j, k)}, \boldsymbol{f}_{(\ell, m, n)}^{(J)} \cdot \boldsymbol{e}_{z}\right\rangle \quad(\text { for } I=4, J=1,2,3), \\
0 \quad \text { (otherwise), }
\end{array}\right.
\end{aligned}
$$

for $i=0,1, \ldots, 2 L+1, j=0,1, \ldots, 2 M+1, k=0,1, \ldots, 2 N+1$, and $\ell=$ $0,1, \ldots, 2 L+1, m=0,1, \ldots, 2 M+1, n=0,1, \ldots, 2 N+1$.

There are eight symmetry modes that are defined by the reflections with respect to three planes $x=0, y=0$ and $z=0$. These modes are expressed as $\hat{\theta}\left(s_{x}, s_{y}, s_{z}\right)$ 
mode, where $s_{x}$ (or $\left.s_{y}, s_{z}\right)=e[o]$ means that $\hat{\theta}$ is an even [odd] function of $x$ (or $\left.y, z\right)$. Therefore, for example in $\hat{\theta}(o, o, e)$ mode, $\hat{\theta}$ is an odd function of $x$ and $y$, and also an even function of $z$. In $\hat{\theta}\left(s_{x}, s_{y}, s_{z}\right)$ mode, the symmetries of $x, y$ and $z$ components of $\hat{\boldsymbol{u}}$ are given by $\left(\bar{s}_{x}, s_{y}, \bar{s}_{z}\right),\left(s_{x}, \bar{s}_{y}, \bar{s}_{z}\right)$ and $\left(s_{x}, s_{y}, s_{z}\right)$, respectively, where $\bar{s}_{x}$ (or $\left.\bar{s}_{y}, \bar{s}_{z}\right)=o[e]$ if $s_{x}\left(\right.$ or $\left.s_{y}, s_{z}\right)=e[o]$.

Because of the symmetry of equations (15)-(18), eigenvalue problem (28) is decomposed into eight eigenvalue problems for $N_{t} / 8$ components of $\boldsymbol{x}$ with the values of $(\ell, m, n)$ in equation (29) corresponding to one of eight symmetry modes in expansions (19) and (20), called $\tilde{\boldsymbol{x}}$. Therefore, we examine the stability of the motionless state for the disturbance of each symmetry mode, similarly to the studies by Edwards (1988) and by Mizushima and Nakamura (2003). The eigenvalue problem for each symmetry mode can be written as

$$
\tilde{A} \tilde{\boldsymbol{x}}=\sqrt{R_{c}} \tilde{B} \tilde{\boldsymbol{x}}
$$

where $\tilde{A}$ and $\tilde{B}$ are matrices of dimension $N_{t} / 8$ obtained by keeping only non-zero components of matrices $A$ and $B$, respectively. Equation (32) is rewritten as

$$
C \tilde{\boldsymbol{x}}=\frac{1}{\sqrt{R_{c}}} \tilde{\boldsymbol{x}}
$$

where $C=\tilde{A}^{-1} \tilde{B}$.

The value of $R_{c}$ for $\hat{\theta}\left(s_{x}, s_{y}, s_{z}\right)$ mode, obtained as the maximum eigenvalue of equation (33), is called $R_{c}^{\left(s_{x}, s_{y}, s_{z}\right)}$ hereinafter. Critical Rayleigh number $R_{c}$ is defined as the minimum of $R_{c}^{\left(s_{x}, s_{y}, s_{z}\right)}$, s for all $s_{x}, s_{y}$ and $s_{z}$. The symmetry mode corresponding to critical Rayleigh number is called the most unstable mode hereinafter. The velocity and temperature fields of most unstable mode are obtained from the eigenvector $\tilde{\boldsymbol{x}}$ of equation (33) for this mode associated with the eigenvalue corresponding to critical Rayleigh number.

\section{Convergence of Numerical Solutions}

The value of $R_{c}^{\left(s_{x}, s_{y}, s_{z}\right)}$ for truncation parameters $(L, M, N)$ is expressed as $R_{c}^{[L, M, N]}$. Relative variations of $R_{c}^{[L, M, N]}$ with the increase in $L, M$ and $N$ by 1 are defined by

$$
\left\{\begin{array}{l}
\varepsilon_{x}^{[L, M, N]}=\frac{\left|R_{c}^{[L+1, M, N]}-R_{c}^{[L, M, N]}\right|}{R_{c}^{[L, M, N]}}, \\
\varepsilon_{y}^{[L, M, N]}=\frac{\left|R_{c}^{[L, M+1, N]}-R_{c}^{[L, M, N]}\right|}{R_{c}^{[L, M, N]}}, \\
\varepsilon_{z}^{[L, M, N]}=\frac{\left|R_{c}^{[L, M, N+1]}-R_{c}^{[L, M, N]}\right|}{R_{c}^{[L, M, N]}},
\end{array}\right.
$$

respectively. Using these variables, maximum relative variation of $R_{c}^{[L, M, N]}$ at $(L, M, N)$, $\varepsilon_{\max }^{[L, M, N]}$, is expressed as

$$
\varepsilon_{\max }^{[L, M, N]}=\max \left(\varepsilon_{x}^{[L, M, N]}, \varepsilon_{y}^{[L, M, N]}, \varepsilon_{z}^{[L, M, N]}\right) .
$$


In the computation of $R_{c}^{\left(s_{x}, s_{y}, s_{z}\right)}$, we start with $L=M=N=3$ or 4 . We then repeat the procedure that $L$ (or $M, N)$ is increased by 1 if $\varepsilon_{\max }^{[L, M, N]}=\varepsilon_{x}^{[L, M, N]}$ (or $\left.\varepsilon_{\max }^{[L, M, N]}=\varepsilon_{y}^{[L, M, N]}, \varepsilon_{z}^{[L, M, N]}\right)$. We observe that $\varepsilon_{\max }^{[L, M, N]}$ tends to decrease to 0 and $R_{c}^{[L, M, N]}$ approaches a certain value, with the increase in $\sqrt{L^{2}+M^{2}+N^{2}}$ under this procedure. Examples of the dependence of $\varepsilon_{\max }^{[L, M, N]}$ on $\sqrt{L^{2}+M^{2}+N^{2}}$ for the most unstable mode are shown in figure 1 for $\left(A_{x}, A_{y}\right)=(0.025,1),(0.1,6)$ and $(0.025,0.025)$. In this figure, roughly exponential decrease in $\varepsilon_{\max }^{[L, M, N]}$ with the increase in $\sqrt{L^{2}+M^{2}+N^{2}}$ is shown.
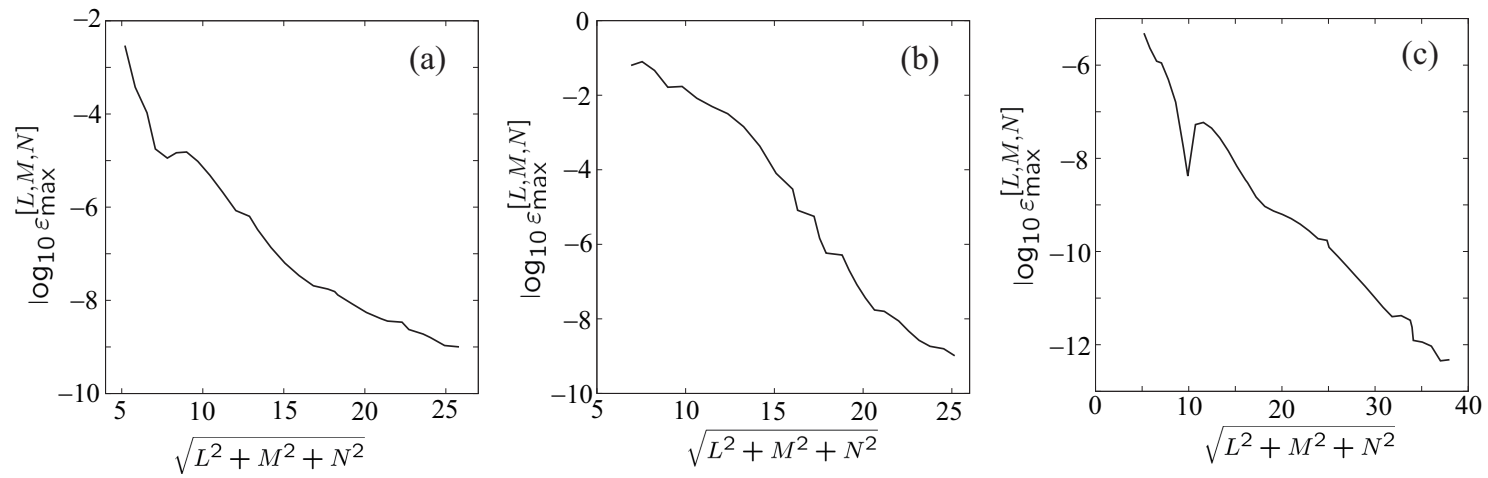

Figure 1. Examples of the dependence of $\varepsilon_{\max }^{[L, M, N]}$ on $\sqrt{L^{2}+M^{2}+N^{2}}$. (a): $\left(A_{x}, A_{y}\right)=(0.025,1) . \hat{\theta}(e, o, e)$ mode. $(\mathrm{b}):\left(A_{x}, A_{y}\right)=(0.1,6)$. $\hat{\theta}(e, e, e)$ mode. (c): $\left(A_{x}, A_{y}\right)=(0.025,0.025) . \hat{\theta}(e, o, e)$ mode.

In most calculations of $R_{c}^{\left(s_{x}, s_{y}, s_{z}\right)}$, the values of $(L, M, N)$ are increased under the above procedure until $N_{t} / 8$ reaches about 7000. For $A_{x}$ and $A_{y}$ satisfying $0.1 \leq A_{x}, A_{y} \leq$ 6 or $0.01 \leq A_{x} \leq 0.1$ and $0.01 \leq A_{y} \leq 1, \varepsilon_{\max }^{[L, M, N]}$ reduces to $10^{-9}$ or less for the final value of $(L, M, N)$ called $\left(L_{m}, M_{m}, N_{m}\right)$. Therefore, the values of $R_{c}^{\left[L_{m}, M_{m}, N_{m}\right]}$ for these $A_{x}$ and $A_{y}$ are regarded as $R_{c}^{\left(s_{x}, s_{y}, s_{z}\right)}$. Here it is noted that the values of $\left(L_{m}, M_{m}, N_{m}\right)$ strongly depend on aspect ratios $A_{x}$ and $A_{y}$ and the symmetry of the mode considered, probably reflecting the difference in the geometrical structures of velocity and temperature fields in the $x, y$ and $z$ directions. This dependence will be discussed in the next section.

Velocity field of $\hat{\theta}\left(s_{x}, s_{y}, s_{z}\right)$ mode denoted by $\hat{\boldsymbol{u}}^{\left(s_{x}, s_{y}, s_{z}\right)}$ is determined from equation (19) by using expansion coefficients $a_{(\ell, m, n)}^{(J)}$ obtained from eigenvector $\tilde{\boldsymbol{x}}$ of equation (33) for $\hat{\theta}\left(s_{x}, s_{y}, s_{z}\right)$ mode associated with the maximum eigenvalue. In order to examine the convergence of velocity fields with the increase in truncation parameters, a measure of difference in velocity fields for different truncation parameters, $\varepsilon_{\boldsymbol{u}}^{[L, M, N]}$, defined by

$$
\varepsilon^{[L, M, N]}=\frac{\max _{x, y, z}\left|\hat{\boldsymbol{u}}^{[\tilde{L}, \tilde{M}, \tilde{N}]}(x, y, z)-\hat{\boldsymbol{u}}^{[L, M, N]}(x, y, z)\right|}{\max _{x, y, z}\left|\hat{\boldsymbol{u}}^{[L, M, N]}(x, y, z)\right|},
$$

is introduced. Here $\hat{\boldsymbol{u}}^{[L, M, N]}$ and $\hat{\boldsymbol{u}}^{[\tilde{L}, \tilde{M}, \tilde{N}]}$ are the velocity fields for truncation parameters $(L, M, N)$ and $(\tilde{L}, \tilde{M}, \tilde{N})$, respectively. Also $(\tilde{L}, \tilde{M}, \tilde{N})$ are the values of truncation parameters after three repetitions of the aforementioned procedure from $(L, M, N)$, 

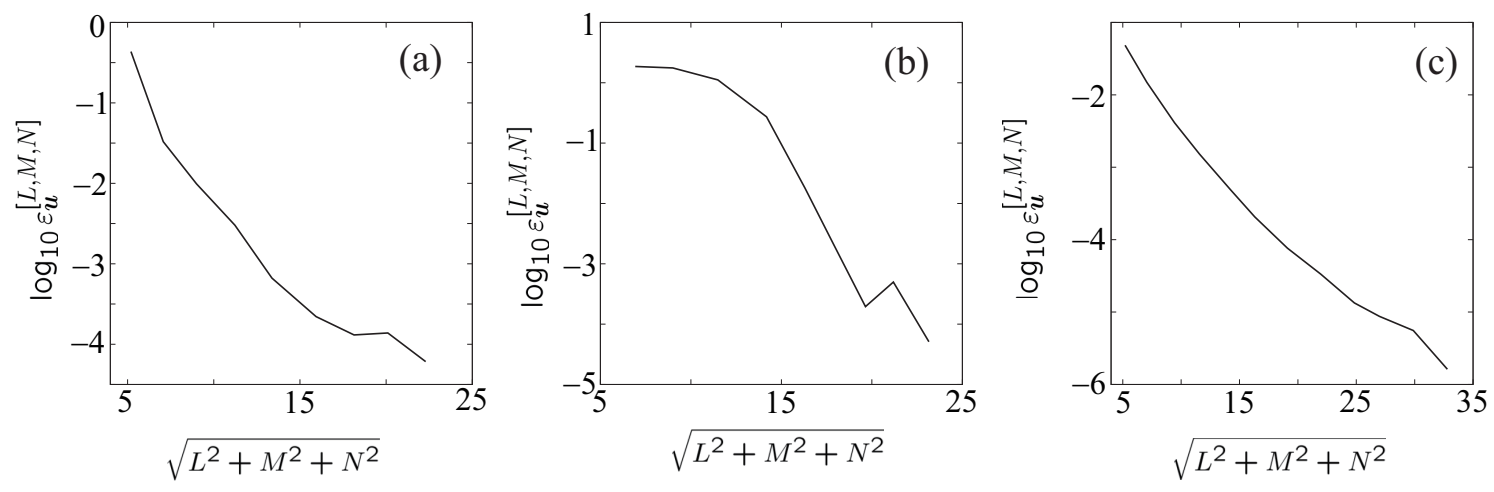

Figure 2. Examples of the dependence of $\varepsilon_{\boldsymbol{u}}^{[L, M, N]}$ on $\sqrt{L^{2}+M^{2}+N^{2}}$. (a): $\left(A_{x}, A_{y}\right)=(0.025,1) . \hat{\theta}(e, o, e)$ mode. $(\mathrm{b}):\left(A_{x}, A_{y}\right)=(0.1,6)$. $\hat{\theta}(e, e, e)$ mode. (c): $\left(A_{x}, A_{y}\right)=(0.025,0.025) . \hat{\theta}(e, o, e)$ mode.

which implies $\tilde{L}+\tilde{M}+\tilde{N}=L+M+N+3 \cdot \varepsilon_{\boldsymbol{u}}^{[L, M, N]}$ tends to decrease exponentially as $\sqrt{L^{2}+M^{2}+N^{2}}$ increases, as illustrated in figure 2 for the most unstable modes for $\left(A_{x}, A_{y}\right)=(0.025,1),(0.1,6)$ and $(0.025,0.025)$. Since the values of $\varepsilon_{\boldsymbol{u}}^{\left[L_{m}, M_{m}, N_{m}\right]}$ are $10^{-4}$ or less for any $A_{x}$ and $A_{y}$ satisfying $0.1 \leq A_{x}, A_{y} \leq 6$ or $0.025 \leq A_{x} \leq 0.1$ and $0.025 \leq A_{y} \leq 1$, the velocity field $\hat{\boldsymbol{u}}^{\left[L_{m}, M_{m}, N_{m}\right]}$ for these $A_{x}$ and $A_{y}$ is regarded as $\hat{\boldsymbol{u}}^{\left(s_{x}, s_{y}, s_{z}\right)}$.

\section{Numerical Results}

In this section, we show the results of numerical examination of $R_{c}$ and the velocity field of most unstable mode both for $A_{x} \leq 1$ and $1 \leq A_{y} \leq 6$ and for $A_{x}=A_{y} \leq 1$.

\subsection{The case of $A_{x} \leq 1$ and $1 \leq A_{y} \leq 6$}

If $A_{x}$ is decreased from 1 with $A_{y}$ being fixed to a value between 1 and 6 , we observe frequent changes of the symmetry of most unstable mode between $\hat{\theta}(e, e, e)$ and $\hat{\theta}(e, o, e)$ modes, as shown in figure 3 . The changes occur more frequently for smaller $A_{x}$ or for larger $A_{y}$.

As found from Table $1, R_{c}$ increases rapidly as $A_{x}$ decreases to 0 , whereas its dependence on $A_{y}$ is relatively weak. Figure 4(a) shows the dependence of $R_{c} A_{x}^{4}$ for $\hat{\theta}(e, o, e)$ and $\hat{\theta}(e, e, e)$ modes on $A_{x}$ for $A_{y}=1$. The changes of the symmetry of most unstable mode occur three times for $A_{x}$ between 0.025 and 0.1 , although the change at the smallest $A_{x}$ is not shown clearly in figure 4(a). The curve of $R_{c} A_{x}^{4}$ defined by $\min \left[R_{c}^{(e, o, e)} A_{x}^{4}, R_{c}^{(e, e, e)} A_{x}^{4}\right]$ in figure 4(a) tends to a finite non-zero value as $A_{x}$ decreases to 0 . Therefore, $R_{c}$ is expected to be approximately proportional to $A_{x}^{-4}$ for sufficiently small $A_{x}$. Figure 4(b) shows the dependence of $R_{c} A_{x}^{4} / \pi^{4}$ on $A_{x}$ for fixed $A_{y}$ of 1,3 or 6. We observe that $R_{c} A_{x}^{4}$ tends to $\pi^{4}$ as $A_{x}$ decreases to 0 irrespective of the value of $A_{y}$. Therefore, $R_{c}$ is well approximated by $\pi^{4} A_{x}^{-4}$ for small $A_{x}$ for any $A_{y}$ of $\mathrm{O}(1)$. This asymptotic behavior of $R_{c}$ will be explained analytically in section 6 . 


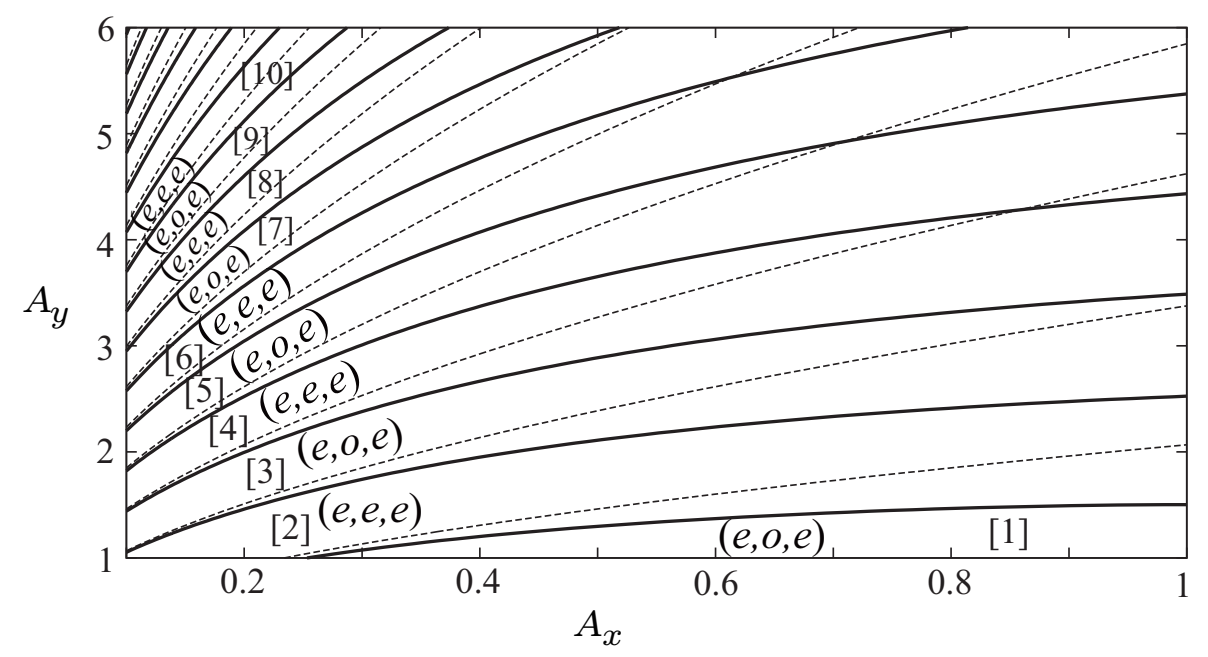

Figure 3. Symmetry of $\hat{\theta}$ of most unstable mode for $0.1 \leq A_{x} \leq 1$ and $1 \leq A_{y} \leq 6$. Solid lines denote the boundaries of the regions of symmetry $\hat{\theta}(e, e, e)$ and $\hat{\theta}(e, o, e)$ in the numerical computations. Broken lines are the boundaries of these regions obtained in the asymptotic analysis. Numbers in [ ] denote the number $N_{v}$ of convection rolls of most unstable mode in the numerical computations.

Table 1. $R_{c}$ and the symmetry of most unstable mode for a few typical aspect ratios with small $A_{x}$.

\begin{tabular}{|c|c|c|c|c|}
\hline$A_{x}$ & $A_{y}$ & $R_{c}$ & symmetry & $\left(L_{m}, M_{m}, N_{m}\right)$ \\
\hline 0.5 & 6 & $9.126607 \times 10^{3}$ & $\hat{\theta}(e, e, e)$ & $(9,12,13)$ \\
\hline 0.5 & 3 & $9.652946 \times 10^{3}$ & $\hat{\theta}(e, e, e)$ & $(9,11,14)$ \\
\hline 0.5 & 1 & $1.497133 \times 10^{4}$ & $\hat{\theta}(e, o, e)$ & $(9,12,13)$ \\
\hline 0.1 & 6 & $1.320239 \times 10^{6}$ & $\hat{\theta}(e, e, e)$ & $(4,19,16)$ \\
\hline 0.1 & 3 & $1.327313 \times 10^{6}$ & $\hat{\theta}(e, e, e)$ & $(5,14,19)$ \\
\hline 0.1 & 1 & $1.398958 \times 10^{6}$ & $\hat{\theta}(e, e, e)$ & $(5,14,19)$ \\
\hline 0.025 & 1 & $2.690256 \times 10^{8}$ & $\hat{\theta}(e, o, e)$ & $(4,11,23)$ \\
\hline
\end{tabular}

The flow pattern of most unstable mode for $A_{x} \ll 1$ and $A_{y}=\mathrm{O}(1)$ is a linear arrangement of several narrow convection rolls in the $y$ direction, as illustrated in figure 5. In this figure, examples of the flow patterns of most unstable $\hat{\theta}(e, e, e)$ and $\hat{\theta}(e, o, e)$ modes for $A_{x} \ll 1$ and $A_{y}=1$ composed of 4 and 5 narrow convection rolls are shown, respectively.

As found from figure 4(a), each change of the symmetry of most unstable mode with the decrease in $A_{x}$ is associated with the increase in the number of convection rolls by one, which causes the decrease in their width-to-height ratio. The dependence of 

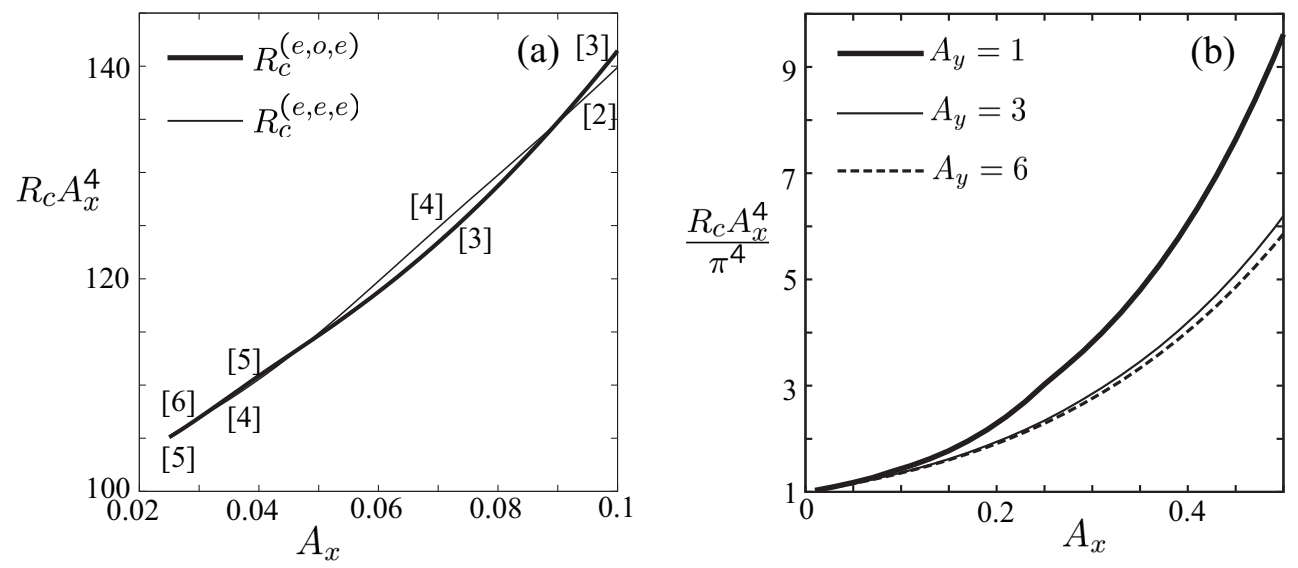

Figure 4. (a): Dependence of $R_{c} A_{x}^{4}$ for $\hat{\theta}(e, o, e)$ and $\hat{\theta}(e, e, e)$ modes on $A_{x} . A_{y}=1$. Numbers in [ ] denote the number $N_{v}$ of convection rolls of these modes. (b): Dependence of $R_{c} A_{x}^{4} / \pi^{4}$ on $A_{x}$ for $A_{y}=1,3$ and 6 .
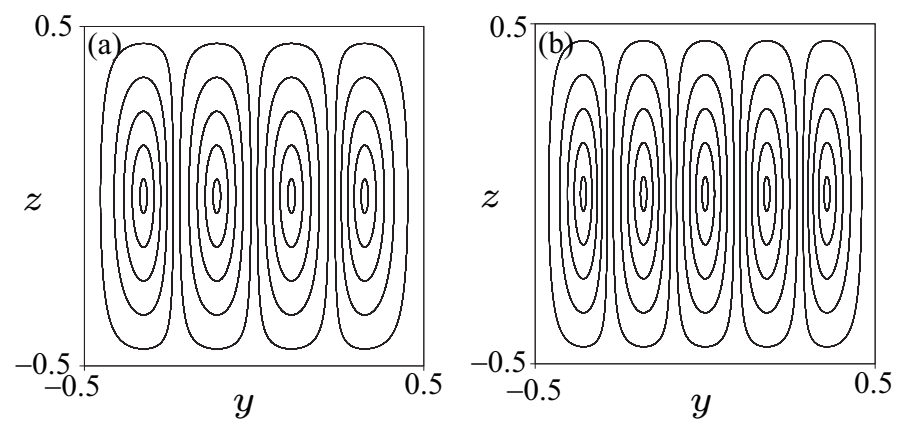

Figure 5. Trajectories of fluid particles on the symmetry plane $x=0 . A_{y}=1$. (a): $A_{x}=0.038, \hat{\theta}(e, e, e)$ mode. (b): $A_{x}=0.025, \hat{\theta}(e, o, e)$ mode.

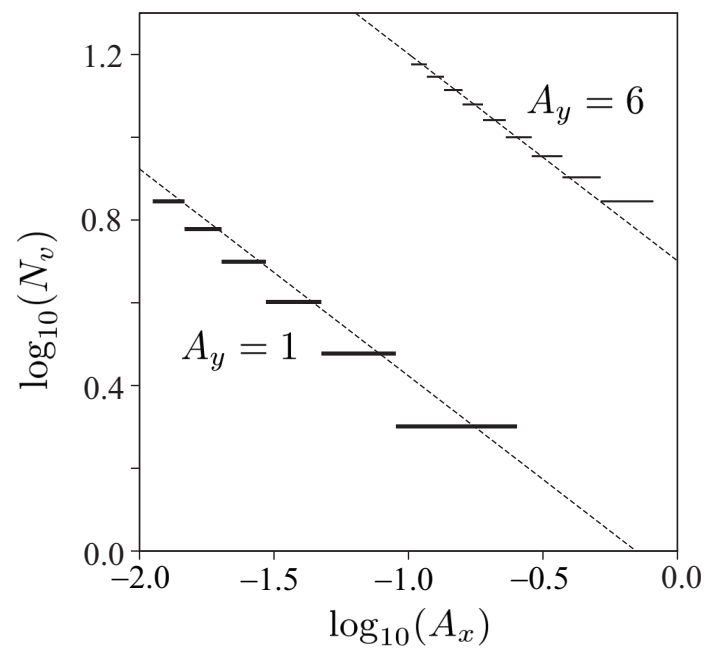

Figure 6. Dependence of the number $N_{v}$ of convection rolls of most unstable mode on $A_{x}$. Thick and thin horizontal line segments denote the range of $A_{x}$ within which the mode of $N_{v}$ convection rolls is the most unstable for $A_{y}=1$ and 6 , respectively. Broken lines with slope $-1 / 2$ denote the value of $N_{v}$ predicted in the asymptotic analysis. 
the number $N_{v}$ of convection rolls of the most unstable mode on $A_{x}$ is shown in figure 6 for $A_{y}=1$ and 6 . At the end points of horizontal line segments of this figure, the changes of most unstable mode occur. Because these points are approximately along broken lines of slope $-1 / 2$, especially for very small $A_{x}$, we can say that $N_{v}$ is roughly proportional to $1 / \sqrt{A_{x}}$ if $A_{x}$ is sufficiently small. That is, the horizontal length (or the width-to-height ratio) of each convection roll of the most unstable mode decreases with the decrease in $A_{x}$ proportionally to $\sqrt{A_{x}}$.
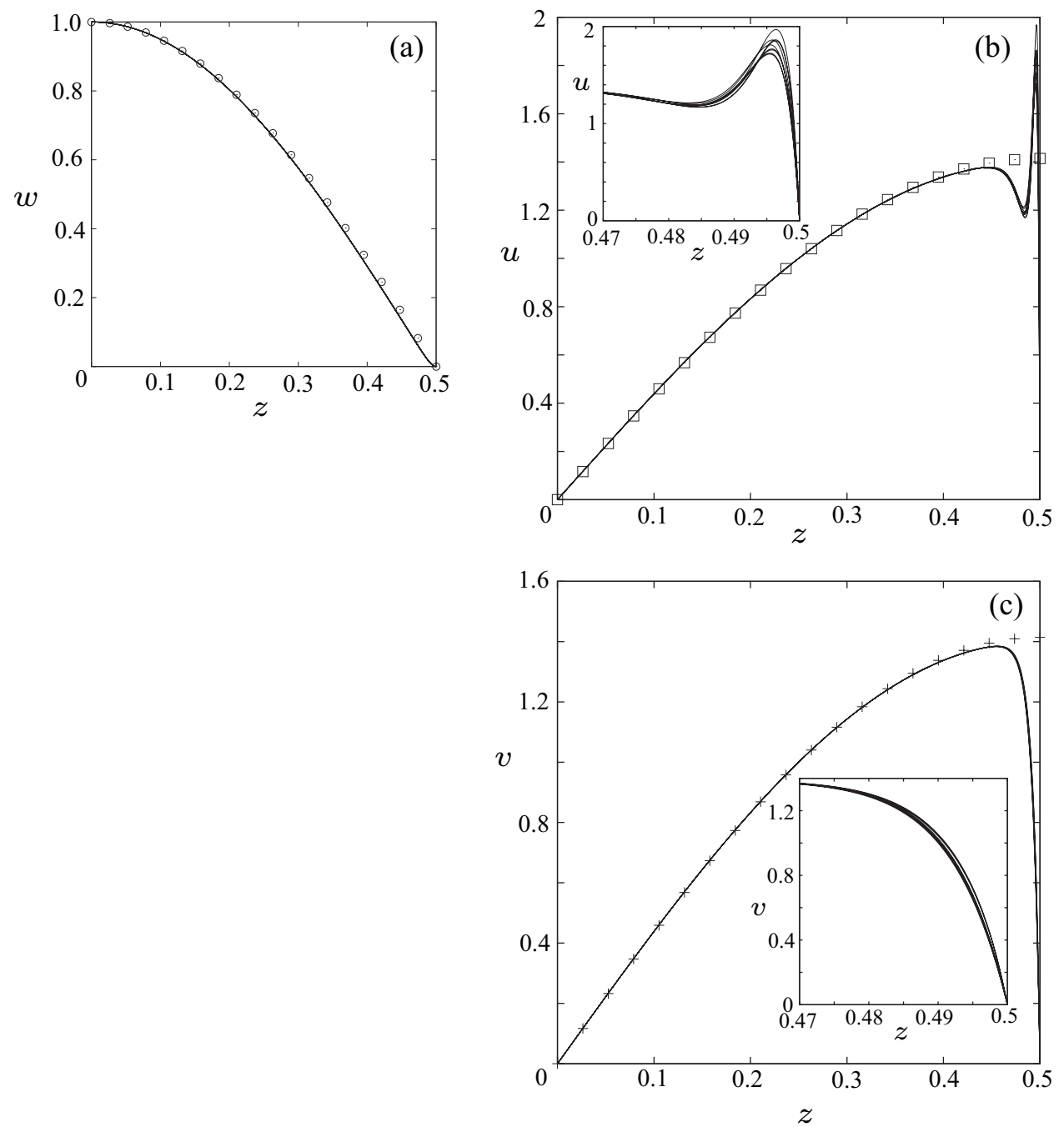

Figure 7. Superposition of the profiles expressing the $z$-dependences of normalized velocity components of most unstable mode at 9 locations of $(x, y)$ where $x=$ $A_{x} / 8, A_{x} / 4$ or $3 A_{x} / 8$ and $y=A_{y} / 8, A_{y} / 4$ or $3 A_{y} / 8 . \quad A_{x}=0.025$ and $A_{y}=1$. (a): Profiles of normalized $w$. (b): Profiles of normalized $u$. (c): Profiles of normalized $v$. In (b) and (c), magnifications of the profiles in the wall layer are shown in the insets. $\square,+$ and $\circ$ denote the profiles of normalized $u, v$ and $w$, respectively, obtained in the asymptotic analysis.

Typical velocity profiles of the $x, y$ and $z$ components of $\hat{\boldsymbol{u}}, u, v$ and $w$, of most unstable mode observed for $A_{x} \ll 1$ and $A_{y}=\mathrm{O}(1)$ are shown in figures 7,8 and 9 . 
Figure 7 shows the normalized profiles expressing the $z$-dependences of these velocity components at 9 locations of $(x, y)$ for $A_{x}=0.025$ and $A_{y}=1$. In figure $7(\mathrm{a}), 9$ profiles of the $w$ normalized so that $w$ is 1 at $z=0$ are shown. Since these profiles are not distinguishable, the profile of normalized $w$ is almost independent of $(x, y)$.

In figure $7(\mathrm{~b}), u$ is normalized so that its value at $z=0.25$ is 1 . The profiles of normalized $u$ at 9 locations of $(x, y)$ are almost the same except in thin regions near the top and bottom walls. We call these regions (about $0.45<z \leq 0.5$ in figure 7 (b)) wall layers hereinafter. As $z$ increases from 0 , all the normalized $u$ increase monotonically from 0 , and approach a local maximum close to 1.4 near the outer edge of the wall layer. In the wall layer, however, the dependence of the profiles of normalized $u$ on $(x, y)$ is observed. As $z$ increases from the outer edge of the wall layer, about 0.45 in figure 7 (b), each of the normalized $u$ first decreases to about 1.2, then increases to a maximum between 1.7 and 2.0, and finally rapidly decreases to 0 .

Figure 7(c) shows the profiles of $v$ normalized in the same way as $u$ at 9 locations of $(x, y)$. Similarly to the profiles of normalized $u$, the profiles of normalized $v$ are almost independent of $(x, y)$ except in the wall layers. As $z$ increases from 0 , all the normalized $v$ monotonically increase to a value around 1.4 near the outer edge of the wall layer. However, within the wall layer, the normalized $v$ is weakly dependent on $(x, y)$ and rapidly decreases to 0 as $z$ approaches 0.5 .

For $A_{x} \ll 1$ and $A_{y}=\mathrm{O}(1)$, because of the rapid variations of $u$ and $v$ in the wall layers shown in figures 7 (b) and (c), large truncation parameter $N$ in the $z$ direction is required for the computation with high accuracy. It is also found that the width of the wall layers, which is about 0.05 for $A_{x}=0.025$, decreases with the decrease in $A_{x}$. Therefore, larger $N_{m}$ is necessary in the numerical computations for smaller $A_{x}$, as illustrated in Table 1.

The $x$-dependences of normalized $u, v$ and $w$ of most unstable mode at 9 locations of $(y, z)$ outside the wall layers for $A_{x}=0.025$ and $A_{y}=1$ are shown in figure 8 . Here $u$ is normalized so that it is 1 at $x=A_{x} / 4$, whereas $v$ and $w$ are normalized so that they are 1 at $x=0$. Because the 9 lines of profiles of each velocity component are not distinguishable, the profiles of normalized $u, v$ and $w$ are almost independent of $y$ and $z$ outside the wall layers. Normalized $v$ and $w$ take a maximum at $x=0$ and monotonically decrease to 0 as side walls $x= \pm A_{x} / 2$ are approached, whereas normalized $u$ takes a maximum near $x= \pm A_{x} / 4$.

Figure 9 shows the $y$-dependences of normalized $u, v$ and $w$ of most unstable mode at 9 locations of $(x, z)$ outside the wall layers for $A_{y}=1$. Here the profiles of each component of velocity are normalized so that their maxima in the region of $0 \leq y \leq 0.5$ is 1 . The 9 lines of profiles of each velocity component are again not distinguishable, which suggests that the profiles of normalized $u, v$ and $w$ are almost independent of $x$ and $z$ outside the wall layers. For $A_{x}=0.038, \hat{\theta}(e, e, e)$ mode is most unstable, in which $u$ and $w$ are even functions of $y$ as shown in figures 9(c) and (a), whereas $v$ is an odd function of $y$ as shown in figure $9(\mathrm{e})$. However, for $A_{x}=0.025, \hat{\theta}(e, o, e)$ mode is most unstable, which causes the opposite parity of velocity components with respect to $y=0$, 


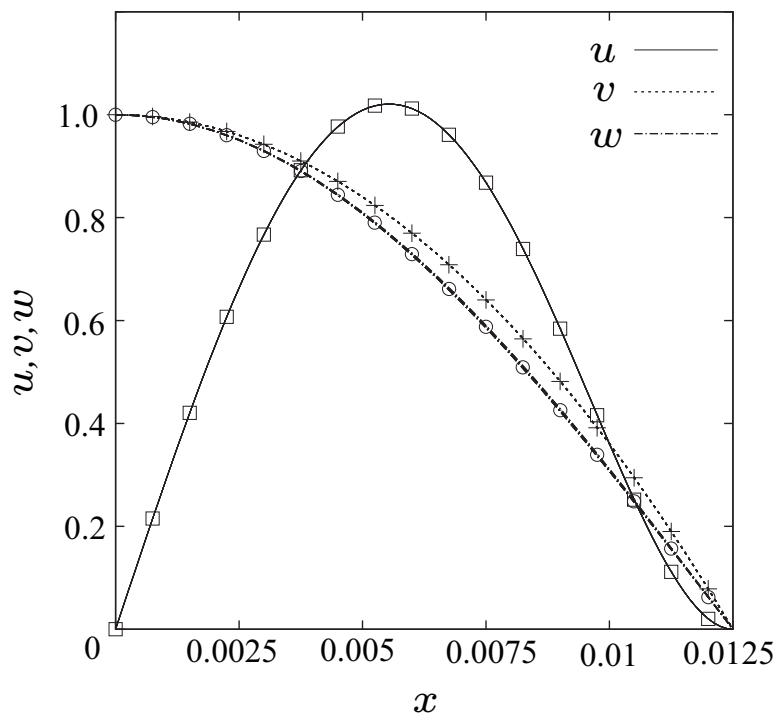

Figure 8. Superposition of the profiles expressing the $x$-dependences of normalized $u$, $v$ and $w$ of most unstable mode at 9 locations of $(y, z)$ where $y=A_{y} / 8, A_{y} / 4$ or $3 A_{y} / 8$ and $z=1 / 8,1 / 4$ or $3 / 8 . A_{x}=0.025$ and $A_{y}=1 . \square,+$ and $\circ$ denote the profiles of normalized $u, v$ and $w$, respectively, obtained in the asymptotic analysis.
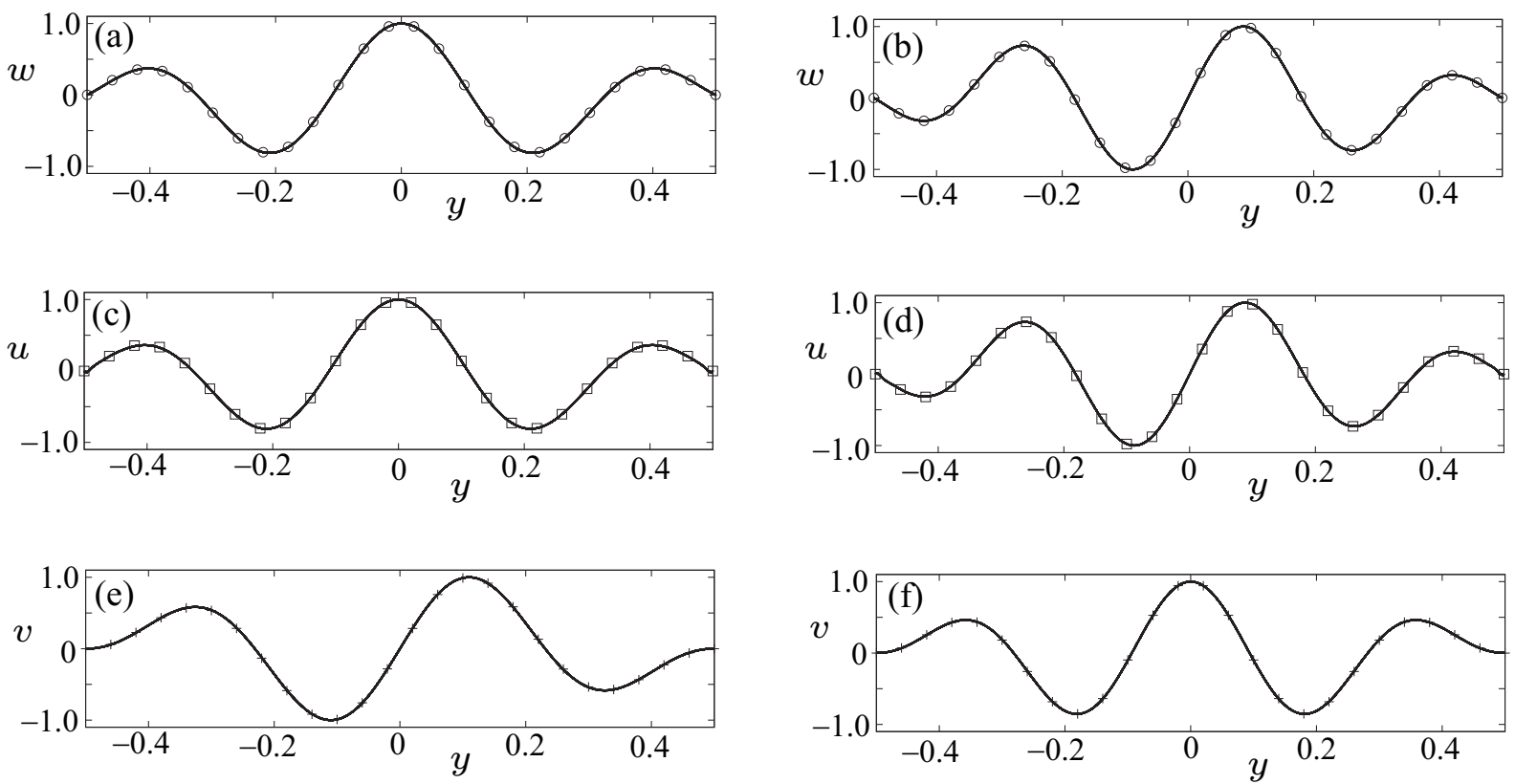

Figure 9. Superposition of the profiles expressing the $y$-dependences of normalized velocity components of most unstable mode at 9 locations of $(x, z)$ where $x=$ $A_{x} / 8, A_{x} / 4$ or $3 A_{x} / 8$ and $z=1 / 8,1 / 4$ or $3 / 8 . A_{y}=1$. (a), (c) and (e): $A_{x}=0.038$, (b), (d) and (f): $A_{x}=0.025$. (a) and (b): Profiles of normalized $w$. (c) and (d): Profiles of normalized $u$. (e) and (f): Profiles of normalized $v . \square,+$ and $\circ$ denote the profiles of normalized $u, v$ and $w$, respectively, obtained in the asymptotic analysis. 
as found from figures $9(\mathrm{~b}),(\mathrm{d})$ and (f). The vertical flows in the flow patterns shown in figure 5 are weaker near the shorter side walls $y= \pm A_{y} / 2$ than those near the central plane $y=0$, as found from figures $9(\mathrm{a})$ and (b). The same tendency is observed also for $u$ and $v$. It is also noted that the profiles of normalized $u$ and $w$ are almost the same, as found from figures $9(\mathrm{a})-(\mathrm{d})$.

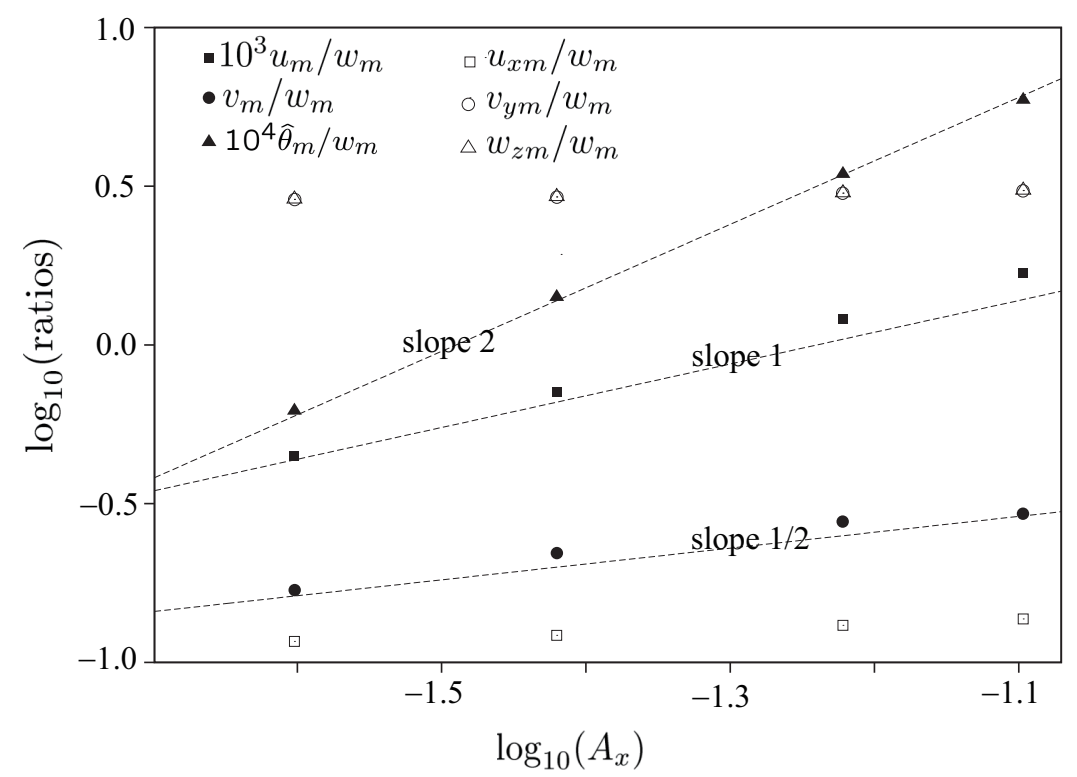

Figure 10. Dependences of the ratios of $u_{m}, v_{m}, \hat{\theta}_{m}, u_{x m}, v_{y m}$ and $w_{z m}$ to $w_{m}$ on $A_{x} . A_{y}=1$. Broken lines are the lines of slopes 2,1 and $1 / 2$.

The ratios of representative magnitudes of $u, v, w$ and $\hat{\theta}$ or their spatial derivatives of the most unstable mode for $A_{x} \ll 1$ and $A_{y}=\mathrm{O}(1)$ are also examined. Figure 10 shows the dependences of the ratios of $u_{m}, v_{m}, \hat{\theta}_{m}, u_{x m}, v_{y m}$ and $w_{z m}$ to $w_{m}$ on $A_{x}$ for $A_{y}=1$, where $u_{m}, v_{m}, w_{m}, \hat{\theta}_{m}, u_{x m}, v_{y m}$ and $w_{z m}$ are the values of $|u|,|v|,|w|,|\hat{\theta}|,|\partial u / \partial x|,|\partial v / \partial y|$ and $|\partial w / \partial z|$ averaged over the $x, y$ and $z$ in the cavity, respectively. Because $u_{m}$ is much smaller than $v_{m}$ and $w_{m}$, the assumption of finite roll adopted by Davis (1967) is a good approximation for $A_{x} \ll 1$ and $A_{y}=\mathrm{O}(1)$. We also find that $u_{m} / w_{m}$, $v_{m} / w_{m}$ and $\hat{\theta}_{m} / w_{m}$ decrease with the decrease in $A_{x}$ proportionally to $A_{x}, \sqrt{A_{x}}$ and $A_{x}^{2}$, respectively. However, $u_{x m} / w_{m}, v_{y m} / w_{m}$ and $w_{z m} / w_{m}$ are almost independent of $A_{x}$. These dependences of several ratios on $A_{x}$ will be used in the asymptotic analysis of section 6 .

\subsection{The case of $A_{x}=A_{y}=A<1$}

In this subsection, we consider the onset of thermal convection in the cavity of a square base with $A_{x}=A_{y}=A<1$. For these aspect ratios, both $\hat{\theta}(e, o, e)$ and $\hat{\theta}(o, e, e)$ modes are most unstable. Critical Rayleigh number $R_{c}$ increases rapidly as $A$ decreases to 0 , as illustrated in Table 2. Moreover, $R_{c}$ is well approximated by $25 \pi^{4} A^{-4}$ for sufficiently 
Table 2. $R_{c}$ and the symmetry of most unstable mode for a few aspect ratios satisfying $A_{x}=A_{y}=A<1$.

\begin{tabular}{|c|c|c|c|}
\hline$A$ & $R_{c}$ & symmetry & $\left(L_{m}, M_{m}, N_{m}\right)$ \\
\hline 0.5 & $4.941374 \times 10^{4}$ & $\hat{\theta}(e, o, e)$ & $(11,9,15)$ \\
\hline 0.1 & $2.454351 \times 10^{7}$ & $\hat{\theta}(e, o, e)$ & $(8,7,25)$ \\
\hline 0.025 & $6.237110 \times 10^{9}$ & $\hat{\theta}(e, o, e)$ & $(5,7,37)$ \\
\hline
\end{tabular}

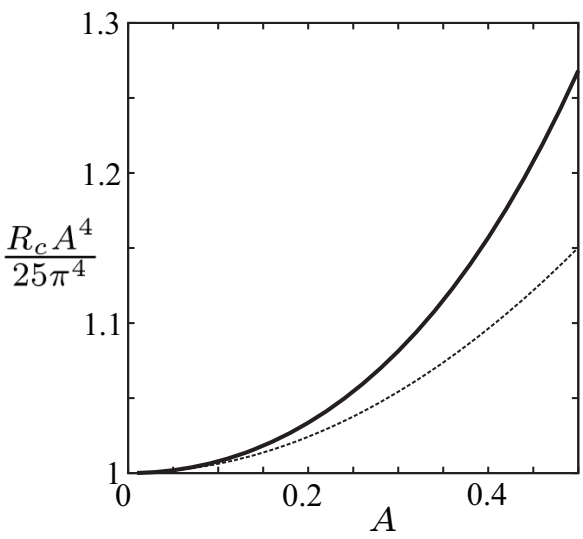

Figure 11. Dependence of $R_{c} A^{4} /\left(25 \pi^{4}\right)$ on $A$. Solid line denotes the numerical result. Broken line is the result of the asymptotic analysis.

small $A$, as found from the solid line in figure 11. This asymptotic behavior of $R_{c}$ will be explained analytically in section 6 .

The flow pattern of most unstable $\hat{\theta}(e, o, e)[\hat{\theta}(o, e, e)]$ mode for small $A$ is composed of only one convection roll whose width-to-height ratio is small, and whose axis is parallel to the $x$ axis $[y$ axis]. An example of this flow pattern for $\hat{\theta}(e, o, e)$ mode is shown in figure 12 for $A=0.0625$. In this figure, the trajectories starting from non-zero $x$ are not on the planes of constant $x$ but are slightly curved if they are projected onto the $x y$ plane. The result suggesting the flow pattern of most unstable mode composed of a narrow convection roll for small $A$ was obtained also by Davis (1967) under the assumption of finite roll.

Typical profiles of the velocity components of most unstable $\hat{\theta}(e, o, e)$ mode for $A \ll 1$ are shown in figure 13. Figure 13(a) shows the $z$-dependences of normalized $u$, $v$ and $w$ at 9 locations of $(x, y)$. Here $u$ and $v$ are normalized so that their values at $z=0.25$ is 1 , whereas $w$ is normalized so that it is 1 at $z=0$. Since the 9 dottedbroken lines in this figure are not distinguishable, the profile of normalized $w$ is almost independent of $(x, y)$.

The 9 profiles of normalized $u$ are almost the same except in thin regions near the 


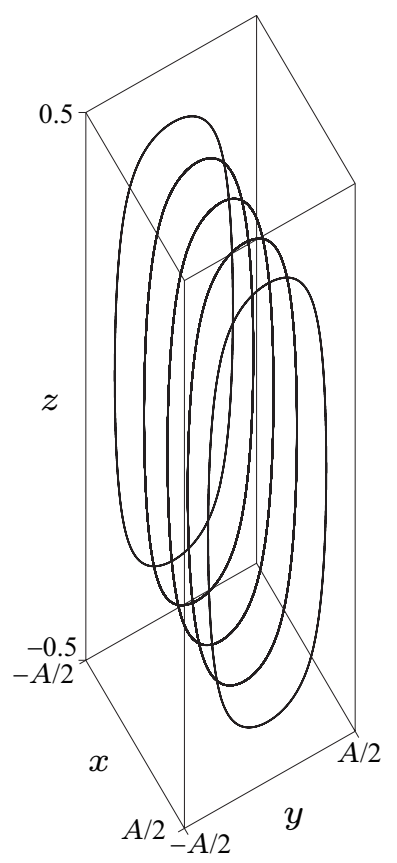

Figure 12. Trajectories of fluid particles starting from 5 points on the line of $y=0$ and $z=0.4$ in the velocity field of most unstable $\hat{\theta}(e, o, e)$ mode. $A=0.0625$.

top and bottom walls, as found from figure 13(a). We again call these regions (about $0.43<z \leq 0.5$ in figure 13(a)) wall layers. With the increase in $z$ from 0 , all the normalized $u$ increases monotonically and approaches a local maximum around 1.4 as $z$ approaches the outer edge of the wall layer. Within the wall layers, however, the dependence of the profiles of normalized $u$ on $(x, y)$ is observed. With the increase in $z$ from the outer edge of the wall layer to 0.5 , each of these profiles first decreases to a local minimum, then takes a maximum at $z$ around 0.49 , and finally decreases rapidly to 0 .

Outside the wall layer, the broken curves of normalized $v$ are hidden behind the solid curves of normalized $u$ in figure 13(a). Therefore, the profiles of normalized $v$ outside the wall layer are very close to the profiles of normalized $u$, and are almost independent of $(x, y)$. However, the normalized $v$ in the wall layer is weakly dependent on $(x, y)$, as found from the inset of this figure.

Because of the rapid variations of $u$ and $v$ in the wall layers shown in figure 13(a), large truncation parameter $N$ in the $z$ direction is again required for the computation of high accuracy for $A \ll 1$. It is also found that the width of the wall layers, which is about 0.07 for $A=0.0625$, decreases as $A$ decreases. Therefore, for smaller $A$, larger $N_{m}$ is necessary for the numerical computations with sufficient accuracy, as illustrated in Table 2.

The $x$-dependences of $u, v$ and $w$ at 9 locations of $(y, z)$ outside the wall layers are shown in figure 13(b). Here $u$ is normalized so that it is 1 at $x=A / 4$, whereas $v$ and $w$ are normalized so that they are 1 at $x=0$. The profiles of the normalized $w$ are almost 

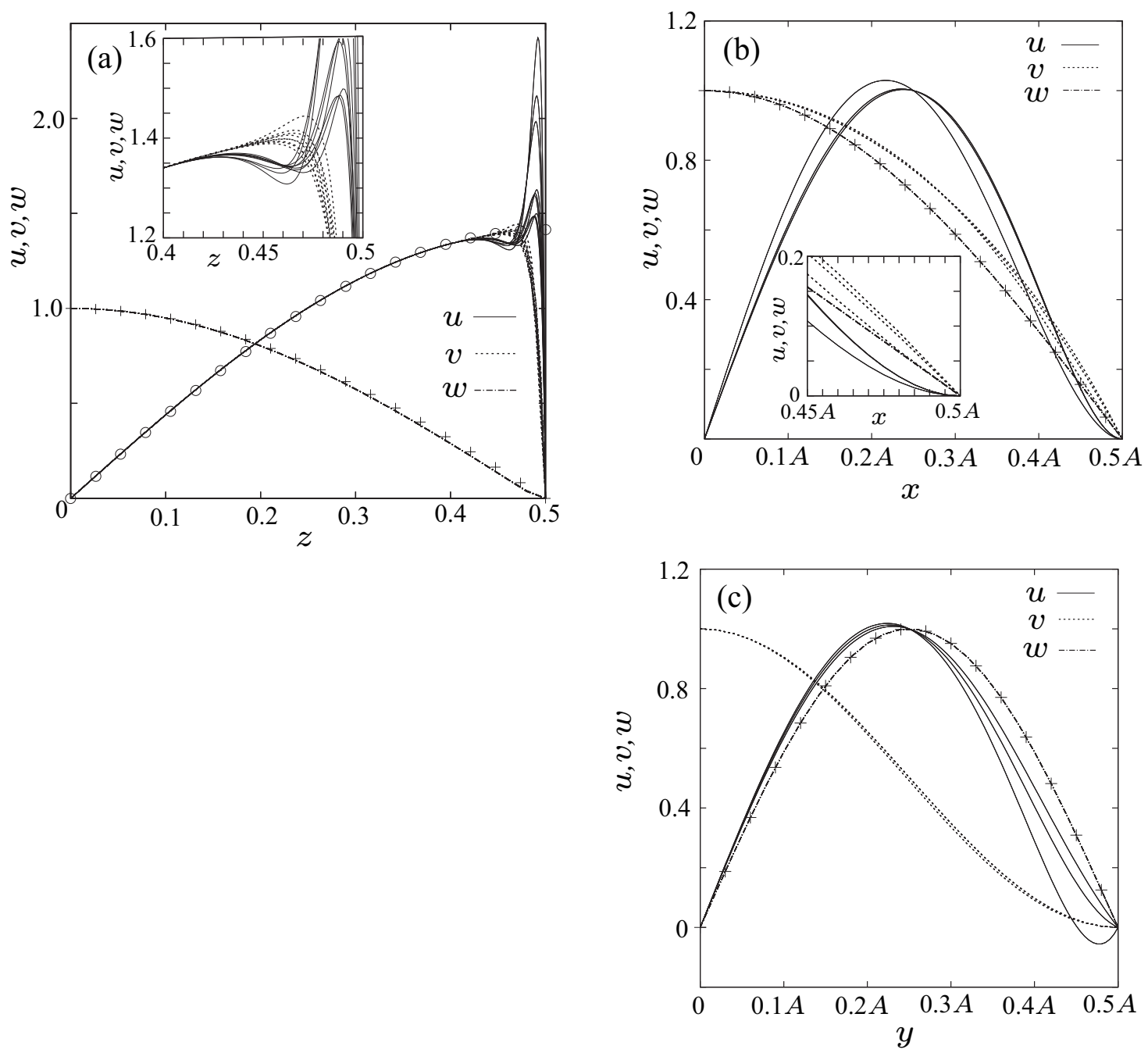

Figure 13. Superposition of profiles expressing the normalized velocity components of most unstable mode at several locations. $A=0.0625$. (a): $z$-dependences of normalized $u, v$ and $w$ at 9 locations of $(x, y)$ where $x=A / 8, A / 4$ or $3 A / 8$ and $y=A / 8, A / 4$ or $3 A / 8$. A magnification of the profiles in the wall layer is shown in the inset. (b): $x$-dependences of normalized $u, v$ and $w$ at 9 locations of $(y, z)$ where $y=A / 8, A / 4$ or $3 A / 8$ and $z=1 / 8,1 / 4$ or $3 / 8$. A magnification of the profiles is shown in the inset. (c): $y$-dependences of normalized $u, v$ and $w$ at 9 locations of $(x, z)$ where $x=A / 8, A / 4$ or $3 A / 8$ and $z=1 / 8,1 / 4$ or $3 / 8$. Symbols + and $\circ$ denote the profiles of normalized $w$ and normalized $u$ and $v$ obtained in the asymptotic analysis, respectively.

independent of $y$ and $z$. However, three curves of profiles are observed for both the normalized $u$ and $v$ (three curves of normalized $v$ are shown more clearly in the inset of figure 13(b)). These curves correspond to the profiles at the locations of three different $y$ 's because the profiles at the locations of same $y$ are not distinguishable even if $z$ is different. This observation suggests that the profiles of normalized $u$ and $v$ vary with $y$ but are independent of $z$.

Figure 13(c) shows the $y$-dependences of $u, v$ and $w$ at 9 locations of $(x, z)$ outside 
the wall layers. Here $u$ and $w$ are normalized so that they are 1 at $y=A / 4$, whereas $v$ is normalized so that it is 1 at $y=0$. We first find that the profiles of normalized $w$ are almost independent of $(x, z)$. However, three curves of normalized $u$ for different $x$ are observed in figure 13(c) because the profiles for the same $x$ are not distinguishable even if $z$ is different. Therefore, it is suggested that the normalized $u$ depends on $x$ but is independent of $z$. The existence of a reverse flow region near $y=A / 2$ in one of three curves of normalized $u$ implies a little complicated flow field near the side walls of $y= \pm A / 2$. The profiles of normalized $v$ also depend only on $x$, although it is not easy to recognize this weak dependence in figure 13(c).

The ratios of $u_{m}, v_{m}, w_{m}, \hat{\theta}_{m}, u_{x m}, v_{y m}$ and $w_{z m}$, introduced in section 5.1 , of the most unstable mode are examined also for $A \ll 1$. Figure 14 shows the dependences of the ratios of $u_{m}, v_{m}, \hat{\theta}_{m}, u_{x m}, v_{y m}$ and $w_{z m}$ to $w_{m}$ on $A$. Because $u_{m}$ is much smaller than $v_{m}$ and $w_{m}$, the assumption of finite roll is a good approximation also for $A \ll 1$. We also find that both $u_{m} / w_{m}$ and $v_{m} / w_{m}$ decrease with the decrease in $A$ proportionally to $A$. However, $\hat{\theta}_{m} / w_{m}$ decreases with the decrease in $A$ proportionally to $A^{2}$. The ratios $u_{x m} / w_{m}, v_{y m} / w_{m}$ and $w_{z m} / w_{m}$ are almost independent of $A$. From the above results, we can say that although the dependence of $v_{m} / w_{m}$ on $A$ for $A \ll 1$ is different from its dependence on $A_{x}$ for $A_{x} \ll 1$ and $A_{y}=\mathrm{O}(1)$, the dependences of other ratios on $A$ for $A \ll 1$ are the same as those on $A_{x}$ for $A_{x} \ll 1$ and $A_{y}=\mathrm{O}(1)$.

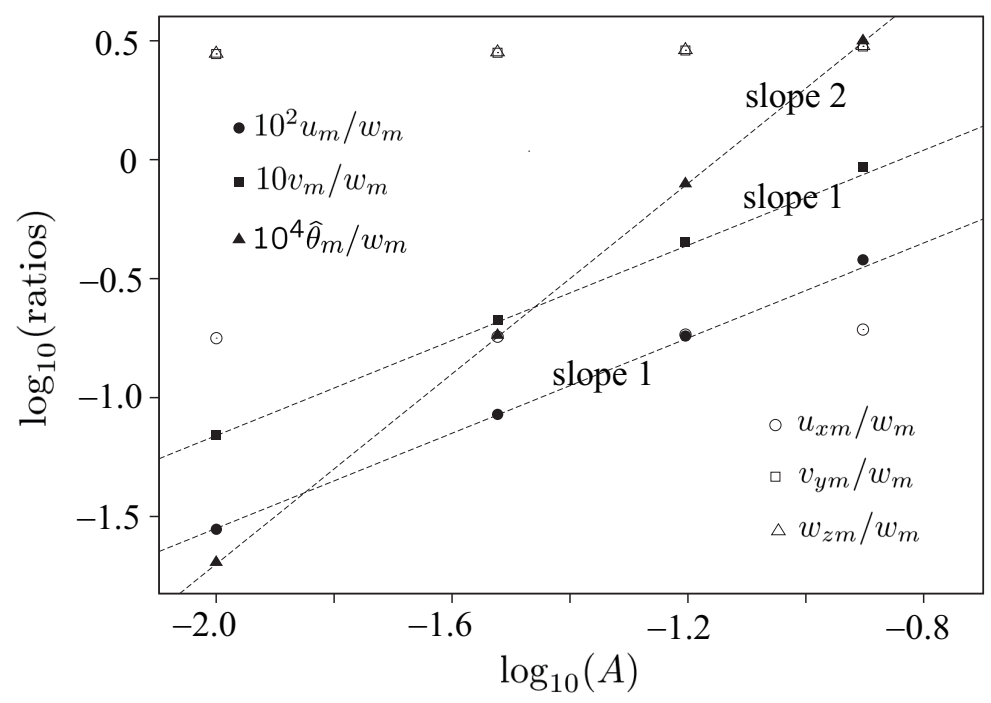

Figure 14. Dependences of the ratios of $u_{m}, v_{m}, \hat{\theta}_{m}, u_{x m}, v_{y m}$ and $w_{z m}$ to $w_{m}$ on A. Broken lines are lines of slopes 1 and 2 .

\section{Asymptotic analysis}

From equations (15)-(17), we can obtain the following equations:

$$
\frac{\partial u}{\partial x}+\frac{\partial v}{\partial y}+\frac{\partial w}{\partial z}=0,
$$




$$
\begin{aligned}
& \frac{\partial}{\partial y} \Delta u-\frac{\partial}{\partial x} \Delta v=0 \\
& \frac{\partial}{\partial z} \Delta u-\frac{\partial}{\partial x} \Delta w-R_{c} \frac{\partial \hat{\theta}}{\partial x}=0 \\
& \frac{\partial}{\partial z} \Delta v-\frac{\partial}{\partial y} \Delta w-R_{c} \frac{\partial \hat{\theta}}{\partial y}=0 \\
& \Delta \hat{\theta}+w=0
\end{aligned}
$$

where $u, v$ and $w$ are the $x, y$ and $z$ components of $\hat{\boldsymbol{u}}$, respectively. Boundary condition (18) is rewritten as

$$
u=v=w=\hat{\theta}=0 \quad \text { on } x= \pm \frac{A_{x}}{2}, \text { on } y= \pm \frac{A_{y}}{2}, \text { and } \text { on } z= \pm \frac{1}{2} .
$$

\subsection{The case of $A_{x} \ll 1$}

Most of the numerical results for $A_{x} \ll 1$ and $A_{y}=\mathrm{O}(1)$ shown in section 5.1 can be explained by using an asymptotic analysis if we adopt a few assumptions based on the numerical results, as will be shown below. In consideration of the numerical results shown in figures 4 and 10, we assume that $u, v, w, \hat{\theta}$ and $R_{c}$ are expressed as

$$
\left\{\begin{array}{l}
u=A_{x}\left(\tilde{u}_{0}+A_{x} \tilde{u}_{1}\right), v=\sqrt{A_{x}}\left(\tilde{v}_{0}+A_{x} \tilde{v}_{1}\right), w=\tilde{w}_{0}+A_{x} \tilde{w}_{1}, \\
\hat{\theta}=A_{x}^{2}\left(\tilde{\theta}_{0}+A_{x} \tilde{\theta}_{1}\right), R_{c}=A_{x}^{-4}\left(\tilde{R}_{0}+A_{x} \tilde{R}_{1}\right),
\end{array}\right.
$$

for small $A_{x}$, where functions $\tilde{u}_{0}, \tilde{v}_{0}, \tilde{w}_{0}, \tilde{\theta}_{0}, \tilde{u}_{1}, \tilde{v}_{1}, \tilde{w}_{1}$ and $\tilde{\theta}_{1}$ are independent of $A_{x}$, and $\tilde{R}_{0}$ and $\tilde{R}_{1}$ are constants independent of $A_{x}$. It is also assumed that these functions depend on

$$
\tilde{x} \equiv \frac{1}{A_{x}} x, \quad \tilde{y} \equiv \frac{1}{\sqrt{A_{x}}} y,
$$

and $z$, because the representative scale of $\hat{\boldsymbol{u}}$ and $\hat{\theta}$ in the $x$ direction is expected to be of $\mathrm{O}\left(A_{x}\right)$, and because the numerical result shown in figure 10 suggests that their representative scale in the $y$ direction is of $\mathrm{O}\left(\sqrt{A_{x}}\right)$.

If we substitute equation (43) into equation (41), we obtain

$$
\tilde{w}_{0}=-\frac{\partial^{2} \tilde{\theta}_{0}}{\partial \tilde{x}^{2}}
$$

at the leading order of $A_{x}$, and

$$
\tilde{w}_{1}=-\frac{\partial^{2} \tilde{\theta}_{1}}{\partial \tilde{x}^{2}}-\frac{\partial^{2} \tilde{\theta}_{0}}{\partial \tilde{y}^{2}}
$$

at the next order of $A_{x}$. Moreover, substituting equation (43) into equations (38)-(40), we obtain

$$
\begin{aligned}
& \frac{\partial^{3} \tilde{v}_{0}}{\partial \tilde{x}^{3}}=0, \\
& \frac{\partial^{3} \tilde{w}_{0}}{\partial \tilde{x}^{3}}+\tilde{R}_{0} \frac{\partial \tilde{\theta}_{0}}{\partial \tilde{x}}=0,
\end{aligned}
$$




$$
\frac{\partial^{3} \tilde{w}_{0}}{\partial \tilde{x}^{2} \partial \tilde{y}}+\tilde{R}_{0} \frac{\partial \tilde{\theta}_{0}}{\partial \tilde{y}}=0
$$

at the leading order, and

$$
\begin{aligned}
& \frac{\partial^{3} \tilde{v}_{1}}{\partial \tilde{x}^{3}}+\frac{\partial^{3} \tilde{v}_{0}}{\partial \tilde{x} \partial \tilde{y}^{2}}-\frac{\partial^{3} \tilde{u}_{0}}{\partial \tilde{x}^{2} \partial \tilde{y}}=0 \\
& \frac{\partial}{\partial \tilde{x}}\left(\frac{\partial^{2} \tilde{w}_{1}}{\partial \tilde{x}^{2}}+\frac{\partial^{2} \tilde{w}_{0}}{\partial \tilde{y}^{2}}\right)+\tilde{R}_{1} \frac{\partial \tilde{\theta}_{0}}{\partial \tilde{x}}+\tilde{R}_{0} \frac{\partial \tilde{\theta}_{1}}{\partial \tilde{x}}=0 \\
& -\frac{\partial^{3} \tilde{v}_{0}}{\partial \tilde{x}^{2} \partial z}+\frac{\partial}{\partial \tilde{y}}\left(\frac{\partial^{2} \tilde{w}_{1}}{\partial \tilde{x}^{2}}+\frac{\partial^{2} \tilde{w}_{0}}{\partial \tilde{y}^{2}}\right)+\tilde{R}_{1} \frac{\partial \tilde{\theta}_{0}}{\partial \tilde{y}}+\tilde{R}_{0} \frac{\partial \tilde{\theta}_{1}}{\partial \tilde{y}}=0,
\end{aligned}
$$

at the next order. Substitution of equation (43) into equation (37) yields

$$
\frac{\partial \tilde{u}_{0}}{\partial \tilde{x}}+\frac{\partial \tilde{v}_{0}}{\partial \tilde{y}}+\frac{\partial \tilde{w}_{0}}{\partial z}=0
$$

at the leading order. From boundary condition (42), we obtain

$$
\begin{array}{llll}
\tilde{u}_{j}=\tilde{v}_{j}=\tilde{w}_{j}=\tilde{\theta}_{j}=0 \quad(j=0,1) & \text { on } & \tilde{x}= \pm \frac{1}{2}, \\
\tilde{u}_{j}=\tilde{v}_{j}=\tilde{w}_{j}=\tilde{\theta}_{j}=0 & (j=0,1) \quad \text { on } & z= \pm \frac{1}{2} .
\end{array}
$$

Using equations (45) and (48), the differential equation

$$
\frac{\partial^{4} \tilde{w}_{0}}{\partial \tilde{x}^{4}}-\tilde{R}_{0} \tilde{w}_{0}=0
$$

for $\tilde{w}_{0}$ is derived. Here, the numerical results shown in figures $7-9$ suggest that for small $A_{x}, w$ can be expressed as a product of a function of $\tilde{x}$, a function of $\tilde{y}$ and a function of $z$ except in the wall layers. Therefore, we assume that $\tilde{w}_{0}$ is expressed as

$$
\tilde{w}_{0}=\tilde{X}_{w}(\tilde{x}) \tilde{Y}_{w}(\tilde{y}) \tilde{Z}_{w}(z),
$$

except in the wall layers, where $\tilde{X}_{w}, \tilde{Y}_{w}$ and $\tilde{Z}_{w}$ are functions of $\tilde{x}, \tilde{y}$ and $z$, respectively. From the equation obtained by substituting equation (57) into equation (56) and boundary condition (54), we find that $\tilde{X}_{w}$ should satisfy

$$
\begin{aligned}
& \frac{d^{4} \tilde{X}_{w}}{d \tilde{x}^{4}}=\tilde{R}_{0} \tilde{X}_{w}, \\
& \tilde{X}_{w}=0 \quad \text { at } \quad \tilde{x}= \pm \frac{1}{2} .
\end{aligned}
$$

The solution to these equations that gives the smallest $\tilde{R}_{0}$ is written as

$$
\tilde{X}_{w}=\cos (\pi \tilde{x}),
$$

for which

$$
\begin{aligned}
& \tilde{w}_{0}=\cos (\pi \tilde{x}) \tilde{Y}_{w}(\tilde{y}) \tilde{Z}_{w}(z), \\
& \tilde{R}_{0}=\pi^{4}
\end{aligned}
$$


are obtained. Moreover, from equations (48), (54) and (59), the following expression of $\tilde{\theta}_{0}$ is derived:

$$
\tilde{\theta}_{0}=\frac{1}{\pi^{2}} \cos (\pi \tilde{x}) \tilde{Y}_{w}(\tilde{y}) \tilde{Z}_{w}(z)
$$

Equation (49) is satisfied by the $\tilde{w}_{0}, \tilde{\theta}_{0}$ and $\tilde{R}_{0}$ obtained above.

The locations of side walls expressed by $y= \pm A_{y} / 2$ correspond to $\tilde{y} \rightarrow \pm \infty$ in the limit of $A_{x} \rightarrow 0$ for fixed $A_{y}$. However, if the boundary condition for $\tilde{y} \rightarrow \pm \infty$ is imposed, the dependence of velocity fields and $R_{c}$ on $A_{y}$ cannot be derived. Therefore, in the following analysis, we use the boundary condition

$$
\tilde{u}_{j}=\tilde{v}_{j}=\tilde{w}_{j}=\tilde{\theta}_{j}=0 \quad(j=0,1) \quad \text { on } \quad \tilde{y}= \pm \tilde{y}_{b},
$$

where $\tilde{y}_{b}=A_{y} /\left(2 \sqrt{A_{x}}\right)$ is assumed to be independent of $A_{x}$. This assumption means that we consider the limit of $A_{x} \rightarrow 0$ with $A_{y}=\mathrm{O}\left(\sqrt{A_{x}}\right)$ instead of $A_{y}=\mathrm{O}(1)$.

Using equations (47) and (53) and boundary conditions (54) and (62), we obtain

$$
\begin{aligned}
& \tilde{u}_{0}=-\frac{1}{\pi}\left[4 \tilde{x}^{3}-3 \tilde{x}+\sin (\pi \tilde{x})\right] \tilde{Y}_{w}(\tilde{y}) \frac{d \tilde{Z}_{w}(z)}{d z}, \\
& \tilde{v}_{0}=\frac{12}{\pi}\left(\tilde{x}^{2}-\frac{1}{4}\right) \frac{d \tilde{Z}_{w}(z)}{d z} \int_{-\tilde{y}_{b}}^{\tilde{y}} \tilde{Y}_{w}\left(\tilde{y}^{\prime}\right) d \tilde{y}^{\prime} .
\end{aligned}
$$

The following equations are derived from boundary conditions (55) and (62) and equations (59), (63) and (64):

$$
\begin{aligned}
& \tilde{Z}_{w}=0 \quad \text { at } \quad z= \pm \frac{1}{2}, \\
& \frac{d \tilde{Z}_{w}}{d z}=0 \quad \text { at } \quad z= \pm \frac{1}{2}, \\
& \tilde{Y}_{w}=0 \quad \text { at } \quad \tilde{y}= \pm \tilde{y}_{b}, \\
& \int_{-\tilde{y}_{b}}^{\tilde{y}_{b}} \tilde{Y}_{w}\left(\tilde{y}^{\prime}\right) d \tilde{y}^{\prime}=0 .
\end{aligned}
$$

However, because we consider only the velocity field outside the wall layers, taking account of the $z$-dependences of $u, v$ and $w$ in the numerical computation shown in figure 7 , we use only equation (65) as the boundary condition for $\tilde{Z}_{w}$ at $z= \pm 1 / 2$.

At the next order of $A_{x}$, from equations (46), (51), (54) and (59)-(61), we obtain

$$
\begin{aligned}
& \tilde{\theta}_{1}=\cos (\pi \tilde{x}) c_{1}(\tilde{y}, z) \\
& +\frac{1}{8 \pi^{5}}\left[2-\frac{\cosh (\pi \tilde{x})}{\cosh (\pi / 2)}-2 \tilde{x} \sin (\pi \tilde{x})\right]\left(\tilde{R}_{1} \tilde{Y}_{w}+2 \pi^{2} \frac{d^{2} \tilde{Y}_{w}}{d \tilde{y}^{2}}\right) \tilde{Z}_{w} \\
& \tilde{w}_{1}=\left[\pi^{2} c_{1}(\tilde{y}, z)+\frac{\tilde{R}_{1}}{2 \pi^{4}} \tilde{Y}_{w} \tilde{Z}_{w}\right] \cos (\pi \tilde{x}) \\
& +\frac{1}{8 \pi^{3}}\left[\frac{\cosh (\pi \tilde{x})}{\cosh (\pi / 2)}-2 \tilde{x} \sin (\pi \tilde{x})\right]\left(\tilde{R}_{1} \tilde{Y}_{w}+2 \pi^{2} \frac{d^{2} \tilde{Y}_{w}}{d \tilde{y}^{2}}\right) \tilde{Z}_{w}
\end{aligned}
$$


where $c_{1}$ is a function of $\tilde{y}$ and $z$. Using equations (52), (59)-(61), (64), (69) and (70), we obtain the following equation for $\tilde{Y}_{w}$ and $\tilde{Z}_{w}$ :

$$
\tilde{R}_{1} \frac{d \tilde{Y}_{w}}{d \tilde{y}} \tilde{Z}_{w}+2 \pi^{2} \frac{d^{3} \tilde{Y}_{w}}{d \tilde{y}^{3}} \tilde{Z}_{w}-96 \frac{d^{2} \tilde{Z}_{w}}{d z^{2}} \int_{-\tilde{y}_{b}}^{\tilde{y}} \tilde{Y}_{w}\left(\tilde{y}^{\prime}\right) d \tilde{y}^{\prime}=0 .
$$

By the differentiation of equation (71) with respect to $\tilde{y}$, the equation

$$
\tilde{R}_{1} \frac{d^{2} \tilde{Y}_{w}}{d \tilde{y}^{2}} \tilde{Z}_{w}+2 \pi^{2} \frac{d^{4} \tilde{Y}_{w}}{d \tilde{y}^{4}} \tilde{Z}_{w}-96 \tilde{Y}_{w} \frac{d^{2} \tilde{Z}_{w}}{d z^{2}}=0
$$

is derived. From equation (72), we obtain the following two equations for $\tilde{Y}_{w}$ and $\tilde{Z}_{w}$ :

$$
\begin{aligned}
& 2 \pi^{2} \frac{d^{4} \tilde{Y}_{w}}{d \tilde{y}^{4}}+\tilde{R}_{1} \frac{d^{2} \tilde{Y}_{w}}{d \tilde{y}^{2}}-\kappa \tilde{Y}_{w}=0, \\
& 96 \frac{d^{2} \tilde{Z}_{w}}{d z^{2}}-\kappa \tilde{Z}_{w}=0,
\end{aligned}
$$

where $\kappa$ is a constant. Here, in consideration of equation (74) and boundary condition (65), it is reasonable to expect that $\tilde{Z}_{w}$ is expressed as

$$
\tilde{Z}_{w}=\cos (\pi z)
$$

because there is always only one convection cell in the vertical direction in the numerical flow pattern of most unstable mode, as illustrated in figure 5. Substituting equation (75) into equation (74), we obtain the relation $\kappa=-96 \pi^{2}$, using which equation (73) is rewritten as

$$
\frac{d^{4} \tilde{Y}_{w}}{d \tilde{y}^{4}}+\frac{\tilde{R}_{1}}{2 \pi^{2}} \frac{d^{2} \tilde{Y}_{w}}{d \tilde{y}^{2}}+48 \tilde{Y}_{w}=0 .
$$

In the case of $\tilde{y}_{b} \rightarrow \infty$, we can assume that $\tilde{Y}_{w}$ is expressed as

$$
\tilde{Y}_{w}=\cos \left(k_{0} \tilde{y}\right)
$$

where $k_{0}$ is a constant. Substitution of equation (77) into equation (76) yields

$$
\tilde{R}_{1}=2 k_{0}^{2} \pi^{2}+\frac{96 \pi^{2}}{k_{0}^{2}} .
$$

Therefore, $\tilde{R}_{1}$ takes the minimum of

$$
\tilde{R}_{1 \infty} \equiv 16 \sqrt{3} \pi^{2}
$$

when $k_{0}$ is equal to $2 \cdot 3^{\frac{1}{4}}$. However, for finite $\tilde{y}_{b}, \tilde{R}_{1}$ is expected to be larger than $\tilde{R}_{1 \infty}$ because $\tilde{Y}_{w}$ is required to satisfy equations (67) and (68) as well as equation (76). From equations (68), (71) and (75), we obtain the boundary condition given by

$$
\frac{d^{3} \tilde{Y}_{w}}{d \tilde{y}^{3}}+\frac{\tilde{R}_{1}}{2 \pi^{2}} \frac{d \tilde{Y}_{w}}{d \tilde{y}}=0 \quad \text { at } \quad \tilde{y}= \pm \tilde{y}_{b}
$$

Odd and even functions satisfying equation (76) are expressed as

$$
\tilde{Y}_{w}=a_{1} \sin \left(\mu_{1} \tilde{y}\right)+a_{2} \sin \left(\mu_{2} \tilde{y}\right)
$$


and

$$
\tilde{Y}_{w}=b_{1} \cos \left(\mu_{1} \tilde{y}\right)+b_{2} \cos \left(\mu_{2} \tilde{y}\right)
$$

respectively, where

$$
\mu_{j}=\frac{1}{2 \pi} \sqrt{\tilde{R}_{1}-(-1)^{j} \sqrt{\tilde{R}_{1}^{2}-\tilde{R}_{1 \infty}^{2}}} \quad(j=1,2),
$$

and $a_{1}, a_{2}, b_{1}$ and $b_{2}$ are constants. Here, $\mu_{1}$ is larger than $\mu_{2}$, and they satisfy the relations

$$
\begin{aligned}
& \mu_{1}^{2}+\mu_{2}^{2}=\frac{\tilde{R}_{1}}{2 \pi^{2}}, \\
& \mu_{1} \mu_{2}=4 \sqrt{3} .
\end{aligned}
$$

It is noted that $\tilde{R}_{1}$ is larger if the difference between $\mu_{1}$ and $\mu_{2}$ is larger.

Because of boundary condition (67), the following equations should be satisfied:

$$
\begin{aligned}
& a_{1} \sin \left(\mu_{1} \tilde{y}_{b}\right)+a_{2} \sin \left(\mu_{2} \tilde{y}_{b}\right)=0, \\
& b_{1} \cos \left(\mu_{1} \tilde{y}_{b}\right)+b_{2} \cos \left(\mu_{2} \tilde{y}_{b}\right)=0 .
\end{aligned}
$$

Moreover, from boundary condition (79), we obtain

$$
\begin{aligned}
& a_{1} \mu_{2} \cos \left(\mu_{1} \tilde{y}_{b}\right)+a_{2} \mu_{1} \cos \left(\mu_{2} \tilde{y}_{b}\right)=0, \\
& b_{1} \mu_{2} \sin \left(\mu_{1} \tilde{y}_{b}\right)+b_{2} \mu_{1} \sin \left(\mu_{2} \tilde{y}_{b}\right)=0,
\end{aligned}
$$

by using equation (83). The condition for the existence of non-zero $a_{1}$ and $a_{2}$ satisfying equations (85) and (87) is written as

$$
\mu_{1} \tan \left(\mu_{1} \tilde{y}_{b}\right)=\mu_{2} \tan \left(\mu_{2} \tilde{y}_{b}\right)
$$

whereas the condition for the existence of non-zero $b_{1}$ and $b_{2}$ satisfying equations (86) and $(88)$ is written as

$$
\mu_{1} \cot \left(\mu_{1} \tilde{y}_{b}\right)=\mu_{2} \cot \left(\mu_{2} \tilde{y}_{b}\right) .
$$

If a value of $\tilde{y}_{b}$ is given, $\mu_{1}$ and $\mu_{2}$ of odd $\tilde{Y}_{w}$ expressed as equation (80) are obtained as the solution to equations (84) and (89), whereas $\mu_{1}$ and $\mu_{2}$ of even $\tilde{Y}_{w}$ given by equation (81) are the solution to equations (84) and (90). Here, although there are infinite number of pairs of $\mu_{1}$ and $\mu_{2}$ satisfying equations (84) and (89) or (90), we choose the pair of smallest difference so that $\tilde{R}_{1}$ determined from equation (83) is the smallest. For fixed $A_{y}$, the values of $\mu_{1}, \mu_{2}$ and $\tilde{R}_{1}$ for any $A_{x}$ are numerically obtained through the above procedure by using $\tilde{y}_{b}=A_{y} /\left(2 \sqrt{A_{x}}\right)$. The dependence of $\tilde{R}_{1}$ on $A_{x}$ for $A_{y}=1$ is shown in figure $15(\mathrm{a})$. Both for odd and even $\tilde{Y}_{w}, \tilde{R}_{1}$ decreases with the decrease in $A_{x}$, and tends to $\tilde{R}_{1 \infty}$. We also observe a few intersections of the $\tilde{R}_{1}$ curves for even and odd $\tilde{Y}_{w}$, corresponding to the change of most unstable mode between $\hat{\theta}(e, e, e)$ and $\hat{\theta}(e, o, e)$ modes. The $R_{c}$ for $\hat{\theta}(e, e, e)$ and $\hat{\theta}(e, o, e)$ modes in the asymptotic analysis are obtained by substituting $\tilde{R}_{0}=\pi^{4}$ and $\tilde{R}_{1}$ for even and odd $\tilde{Y}_{w}$ into equation (43). The $\tilde{R}_{c} \equiv R_{c} A_{x}^{4} / \pi^{4}$ based on these $R_{c}$, shown in figure $15(\mathrm{~b})$ as thin and thick 

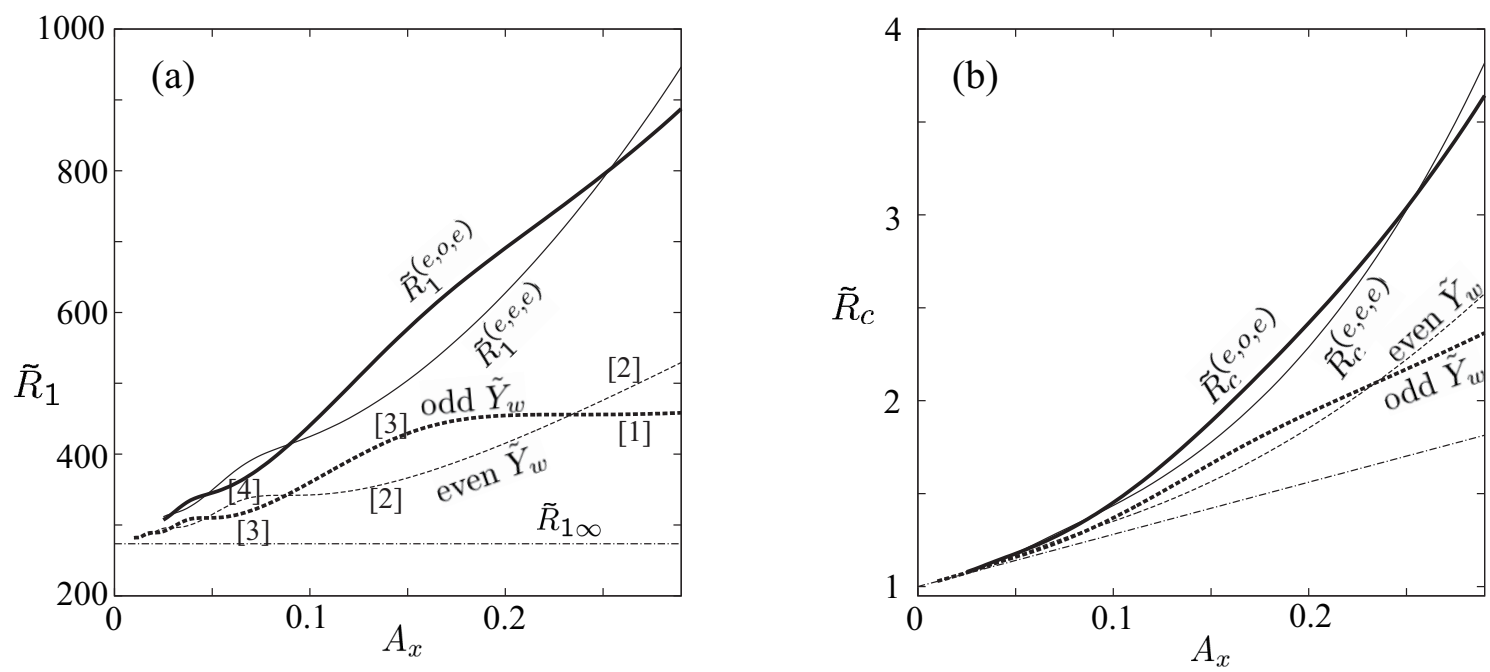

Figure 15. (a): Thick and thin broken lines denote the $\tilde{R}_{1}$ for odd and even $\tilde{Y}_{w}$, respectively. $\quad \tilde{R}_{1 \infty}$ is expressed by a dotted-broken line. Numbers in [ ] denote the number of convection rolls, $N_{v}$. $\tilde{R}_{1}^{(e, o, e)}$ and $\tilde{R}_{1}^{(e, e, e)}$ denoted by thick and thin solid lines are defined by equation (99). $A_{y}=1$. (b): Thick and thin broken lines denote the $\tilde{R}_{c} \equiv R_{c} A_{x}^{4} / \pi^{4}$ computed from equation (101) with the $\tilde{R}_{1}$ for odd and even $\tilde{Y}_{w}$, respectively, whereas dotted-broken line denotes the $\tilde{R}_{c}$ obtained by using $\tilde{R}_{1}=\tilde{R}_{1 \infty}$ in equation (101). $\tilde{R}_{c}^{(e, o, e)}$ and $\tilde{R}_{c}^{(e, e, e)}$ denoted by thick and thin solid lines are defined by equation (100). $A_{y}=1$.

broken lines, decreases to 1 as $A_{x}$ decreases to 0 both for even and odd $\tilde{Y}_{w}$, and is well approximated by the $\tilde{R}_{c}$ computed from $\tilde{R}_{0}=\pi^{4}$ and $\tilde{R}_{1}=\tilde{R}_{1 \infty}$ expressed by a straight dotted-broken line for small $A_{x}$.

The $A_{x}$ 's at the intersections of $\tilde{R}_{1}$ curves for odd and even $\tilde{Y}_{w}$ can be obtained in the asymptotic analysis. That is, if $\tilde{R}_{1}$ 's for odd and even $\tilde{Y}_{w}$ are the same, the $\mu_{1}$ and $\mu_{2}$ for odd $\tilde{Y}_{w}$ agree with those for even $\tilde{Y}_{w}$. Therefore, these $\mu_{1}$ and $\mu_{2}$ are required to satisfy equations (89) and (90) simultaneously. The series of $\mu_{1}$ and $\mu_{2}$ satisfying these equations are written as

$$
\mu_{1}=\frac{\left(N_{b}+1\right) \pi}{\tilde{y}_{b}}, \quad \mu_{2}=\frac{N_{b} \pi}{\tilde{y}_{b}} \quad\left(N_{b}=1,2, \ldots\right)
$$

or

$$
\mu_{1}=\frac{\left(N_{b}+\frac{1}{2}\right) \pi}{\tilde{y}_{b}}, \quad \mu_{2}=\frac{\left(N_{b}-\frac{1}{2}\right) \pi}{\tilde{y}_{b}} \quad\left(N_{b}=1,2, \ldots\right) .
$$

When equation (91) is satisfied, we obtain

$$
\begin{gathered}
\tilde{y}_{b}=\tilde{y}_{b(e o)}^{\left(N_{b}\right)}, \quad \text { where } \quad \tilde{y}_{b(e o)}^{\left(N_{b}\right)} \equiv \sqrt{\frac{N_{b}\left(N_{b}+1\right)}{4 \sqrt{3}} \pi} \quad\left(N_{b}=1,2, \ldots\right), \\
\tilde{R}_{1}=\tilde{R}_{1(e o)}^{\left(N_{b}\right)}, \quad \text { where } \tilde{R}_{1(e o)}^{\left(N_{b}\right)} \equiv \tilde{R}_{1 \infty}\left[1+\frac{1}{2 N_{b}\left(N_{b}+1\right)}\right] \quad\left(N_{b}=1,2, \ldots\right),
\end{gathered}
$$


from equations (83) and (84), whereas

$$
\begin{gathered}
\tilde{y}_{b}=\tilde{y}_{b(o e)}^{\left(N_{b}\right)}, \quad \text { where } \quad \tilde{y}_{b(o e)}^{\left(N_{b}\right)} \equiv \sqrt{\frac{N_{b}^{2}-\frac{1}{4}}{4 \sqrt{3}} \pi \quad\left(N_{b}=1,2, \ldots\right),} \\
\tilde{R}_{1}=\tilde{R}_{1(o e)}^{\left(N_{b}\right)}, \quad \text { where } \quad \tilde{R}_{1(o e)}^{\left(N_{b}\right)} \equiv \tilde{R}_{1 \infty}\left[1+\frac{1}{2\left(N_{b}^{2}-\frac{1}{4}\right)}\right] \quad\left(N_{b}=1,2, \ldots\right),
\end{gathered}
$$

are obtained from the same equations if equation (92) is satisfied. Both of the above two expressions of $\tilde{R}_{1}$ can be rewritten as

$$
\tilde{R}_{1}=\tilde{R}_{1 \infty}\left(1+\frac{\pi^{2}}{8 \sqrt{3} \tilde{y}_{b}^{2}}\right)
$$

for any $N_{b}$.

The $\tilde{y}_{b}$ 's defined by equations (93) or (95), which corresponds to the intersections of $\tilde{R}_{1}$ curves for odd and even $\tilde{Y}_{w}$, increase roughly proportionally with $N_{b}$ for large $N_{b}$. The $\tilde{R}_{1}$ 's at these intersections decrease with the increase in $\tilde{y}_{b}$ or $N_{b}$, and tend to $\tilde{R}_{1 \infty}$ as $\tilde{y}_{b}$ (or $N_{b}$ ) $\rightarrow \infty$, as found from equations (94), (96) and (97). It is also found that the relation $\tilde{y}_{b(o e)}^{\left(N_{b}\right)}<\tilde{y}_{b(e o)}^{\left(N_{b}\right)}<\tilde{y}_{b(o e)}^{\left(N_{b}+1\right)}$ holds for any positive integer $N_{b}$. The values of $\tilde{y}_{b}$ and $\tilde{R}_{1}$ given by equations (93)-(96) for $N_{b}=1,2$ and 3 are shown in Table 3 in ascending order of $\tilde{y}_{b}$. The values of $A_{x}$ based on the above $\tilde{y}_{b}$ for $A_{y}=1$ are also shown in this table to compare with the numerical results.

Table 3. Values of $\tilde{y}_{b}$ and $\tilde{R}_{1}$ given by equations (93)-(96). Values of $A_{x}$ based on this $\tilde{y}_{b}$ and numerically computed $A_{x}$ where the most unstable mode changes for $A_{y}=1$.

\begin{tabular}{|c|c|c|c|c|c|c|}
\hline $\begin{array}{c}(e o) \\
\text { or } \\
(o e)\end{array}$ & $N_{b}$ & $\tilde{y}_{b}$ & $\tilde{R}_{1}$ & $\begin{array}{c}A_{x} \text { based } \\
\text { on } \tilde{y}_{b} \text { for } \\
A_{y}=1\end{array}$ & $\begin{array}{c}A_{x} \text { where the most } \\
\text { unstable mode } \\
\text { changes for } A_{y}=1\end{array}$ & $\begin{array}{c}\text { change of } N_{v} \text { of } \\
\text { most unstable mode } \\
\text { with the increase in } \tilde{y}_{b}\end{array}$ \\
\hline$(o e)$ & 1 & 1.0336 & 455.86 & 0.2340 & 0.2533 & $1 \rightarrow 2$ \\
\hline$(e o)$ & 1 & 1.6879 & 341.89 & 0.0877 & 0.0898 & $2 \rightarrow 3$ \\
\hline$(o e)$ & 2 & 2.3113 & 309.98 & 0.0468 & 0.0474 & $3 \rightarrow 4$ \\
\hline$(e o)$ & 2 & 2.9236 & 296.31 & 0.0292 & 0.0295 & $4 \rightarrow 5$ \\
\hline$(o e)$ & 3 & 3.5306 & 289.14 & 0.0201 & 0.0202 & $5 \rightarrow 6$ \\
\hline$(e o)$ & 3 & 4.1346 & 284.91 & 0.0146 & 0.0147 & $6 \rightarrow 7$ \\
\hline
\end{tabular}

The number of convection rolls, $N_{v}$, agrees with the number of zeros of the profile of $y$-dependence of $w$ in the region of $-A_{y} / 2<y<A_{y} / 2$, as found from the comparison of figures 5 and 9 (a) and (b). The $y$-dependence of normalized $w$ is determined by $\tilde{Y}_{w}$ in the asymptotic analysis. For each $\tilde{y}_{b}$, because the ratios of coefficients, $a_{2} / a_{1}$ and $b_{2} / b_{1}$, 
in equations (80) and (81) are determined from equations (85) and (86) (or equations (87) and (88)), the profiles of even and odd $\tilde{Y}_{w}$ are obtained. On the basis of the number of zeros of these $\tilde{Y}_{w}$, we obtain the results that at $\tilde{y}_{b}=\tilde{y}_{b(e o)}^{\left(N_{b}\right)}$, the change of most unstable mode between the even $\tilde{Y}_{w}$ with $N_{v}=2 N_{b}$ and the odd $\tilde{Y}_{w}$ with $N_{v}=2 N_{b}+1$ occurs, and that at $\tilde{y}_{b}=\tilde{y}_{b(o e)}^{\left(N_{b}\right)}$, the change between the odd $\tilde{Y}_{w}$ with $N_{v}=2 N_{b}-1$ and the even $\tilde{Y}_{w}$ with $N_{v}=2 N_{b}$ occurs. Therefore, as $\tilde{y}_{b}$ increases, the $N_{v}$ of most unstable mode increases by 1 at the above $\tilde{y}_{b}$ 's, as shown in Table 3 . This behavior corresponds to the increase in the $N_{v}$ one by one with the decrease in $A_{x}$ for fixed $A_{y}$, as shown in figures $3,4(\mathrm{a})$ and 15(a). For large $N_{b}$, the $N_{v}$ is approximated by $2 N_{b}$, and both $\tilde{y}_{b(e o)}^{\left(N_{b}\right)}$ and $\tilde{y}_{b(o e)}^{\left(N_{b}\right)}$ are approximated by $\pi N_{b} /\left(2 \cdot 3^{\frac{1}{4}}\right)$. Therefore, we obtain the following approximate expression of $N_{v}$ :

$$
N_{v}=\frac{2 \cdot 3^{\frac{1}{4}} A_{y}}{\pi \sqrt{A_{x}}}
$$

The results of asymptotic analysis obtained in this subsection agree well with the numerical results for small $A_{x}$ shown in section 5.1. That is, in the asymptotic analysis, the boundary lines in the $\left(A_{x}, A_{y}\right)$ plane across which the most unstable mode changes are determined from $\tilde{y}_{b}=\tilde{y}_{b(e o)}^{\left(N_{b}\right)}$ and $\tilde{y}_{b}=\tilde{y}_{b(o e)}^{\left(N_{b}\right)}$. These boundary lines denoted by broken lines in figure 3 agree well with the numerical boundary lines for small $A_{x}$, although their difference is not small for $A_{x}$ around 1 . Moreover, for $A_{y}=1$, quite good agreement between analytical and numerical $A_{x}$ 's where the most unstable mode changes is observed for small $A_{x}$, as found from Table 3 . The relation between $A_{x}$ and $N_{v}$ given by equation (98) is shown in figure 6 by broken lines for $A_{y}=1$ and 6 . We find that the dependence of $N_{v}$ on $A_{x}$ in the numerical computation for small $A_{x}$ is consistent with these lines. It is also found that the one-by-one increase in the $N_{v}$ of most unstable mode with the decrease in $A_{x}$ for fixed $A_{y}$ in the numerical computations is perfectly explained by the asymptotic analysis.

The values of $\tilde{R}_{1}^{(e, e, e)}$ and $\tilde{R}_{1}^{(e, o, e)}$ defined by

$$
\tilde{R}_{1}^{\left(e, s_{y}, e\right)} \equiv \frac{A_{x}^{4} R_{c}^{\left(e, s_{y}, e\right)}-\pi^{4}}{A_{x}} \quad\left(s_{y}=e \text { or } o\right),
$$

obtained in the numerical computation, correspond to the $\tilde{R}_{1}$ in the asymptotic analysis for even and odd $\tilde{Y}_{w}$, respectively. The dependence of $\tilde{R}_{1}^{(e, o, e)}$ and $\tilde{R}_{1}^{(e, e, e)}$ on $A_{x}$ for $A_{y}=1$ is expressed by thick and thin solid lines in figure $15(\mathrm{a})$, respectively. Although they are larger than the $\tilde{R}_{1}$ for odd and even $\tilde{Y}_{w}$, their deviation from this $\tilde{R}_{1}$ decreases as $A_{x}$ decreases, and they tend to $\tilde{R}_{1 \infty}$ as $A_{x} \rightarrow 0$. The dependence of $\tilde{R}_{c}^{(e, e, e)}$ and $\tilde{R}_{c}^{(e, o, e)}$ defined by

$$
\tilde{R}_{c}^{\left(e, s_{y}, e\right)} \equiv R_{c}^{\left(e, s_{y}, e\right)} A_{x}^{4} / \pi^{4} \quad\left(s_{y}=e \text { or } o\right),
$$

obtained in the numerical computation, on $A_{x}$ for $A_{y}=1$ is expressed by solid lines in figure $15(\mathrm{~b})$. The dependence of the analytical result of $\tilde{R}_{c}$ defined by

$$
\tilde{R}_{c} \equiv 1+\frac{A_{x} \tilde{R}_{1}}{\pi^{4}}
$$


with the $\tilde{R}_{1}$ for even and odd $\tilde{Y}_{w}$ on $A_{x}$ for $A_{y}=1$ is shown in figure $15(\mathrm{~b})$ as thin and thick broken lines, respectively, whereas the dependence of the $\tilde{R}_{c}$ with $\tilde{R}_{1}=\tilde{R}_{1 \infty}$ on $A_{x}$ is expressed as a dotted-broken line. Both $\tilde{R}_{c}^{(e, e, e)}$ and $\tilde{R}_{c}^{(e, o, e)}$ are tangent to the line of the $\tilde{R}_{c}$ with $\tilde{R}_{1}=\tilde{R}_{1 \infty}$ in the limit of $A_{x} \rightarrow 0$, and are well approximated by the $\tilde{R}_{c}$ with the $\tilde{R}_{1}$ for even and odd $\tilde{Y}_{w}$ for small $A_{x}$.

The numerical profiles of normalized $u, v$ and $w$ of most unstable mode shown in figures 7-9 also agree well with the profiles obtained in the asymptotic analysis outside the wall layers. That is, the $z$-dependence of normalized $w$ shown in figure 7 (a) is well approximated by the analytical profile of $\tilde{Z}_{w}=\cos (\pi z)$ of equation (75), denoted by $\circ$, except for a slight discrepancy near $z= \pm 0.5$. The $z$-dependences of normalized $u$ and $v$ shown in figures $7(\mathrm{~b})$ and (c) are also close to the analytical profile of $\sqrt{2} \sin (\pi z)$, denoted by $\square$ and + , derived from equations (63), (64) and (75) except in the wall layers. The $x$-dependences of normalized $u, v$ and $w$ outside the wall layers shown in figure 8 are well approximated by the analytical profiles obtained by normalizing the $\tilde{u}_{0}, \tilde{v}_{0}$ and $\tilde{w}_{0}$ given by equations (63), (64) and (59), expressed by $\square,+$ and $\circ$, respectively. The $y$-dependences of normalized $u$ and $w$ shown in figures $9(\mathrm{a})-(\mathrm{d})$ also agree well with the normalized profiles of $\tilde{Y}_{w}$ expressing the $y$-dependence of $\tilde{u}_{0}$ and $\tilde{w}_{0}$ in equations (63) and (59), denoted by $\square$ and $\circ$, both for $\hat{\theta}(e, e, e)$ and $\hat{\theta}(e, o, e)$ modes. Moreover, the $y$-dependences of normalized $v$ shown in figures $9(\mathrm{e})$ and (f) are well approximated by the normalized $y$-dependences of $\tilde{v}_{0}$, denoted by + , obtained by substituting even and odd $\tilde{Y}_{w}$ into equation (64), respectively.

\subsection{The case of $A_{x}=A_{y}=A \ll 1$}

Most of the numerical results for $A \ll 1$ shown in section 5.2 also can be explained by using an asymptotic analysis under a few assumptions based on the numerical results. That is, in consideration of the results shown in figures 11 and 14, we assume that $u, v, w, \hat{\theta}$ and $R_{c}$ are expressed as

$$
\left\{\begin{array}{l}
u=A\left(\hat{u}_{0}+A^{2} \hat{u}_{1}\right), v=A\left(\hat{v}_{0}+A^{2} \hat{v}_{1}\right), w=\hat{w}_{0}+A^{2} \hat{w}_{1}, \\
\hat{\theta}=A^{2}\left(\hat{\theta}_{0}+A^{2} \hat{\theta}_{1}\right), R_{c}=A^{-4}\left(\hat{R}_{0}+A^{2} \hat{R}_{1}\right),
\end{array}\right.
$$

for small $A$, where functions $\hat{u}_{0}, \hat{v}_{0}, \hat{w}_{0}, \hat{\theta}_{0}, \hat{u}_{1}, \hat{v}_{1}, \hat{w}_{1}$ and $\hat{\theta}_{1}$ are independent of $A$, and $\hat{R}_{0}$ and $\hat{R}_{1}$ are constants independent of $A$. It is also assumed that these functions depend on

$$
\hat{x} \equiv \frac{1}{A} x, \quad \hat{y} \equiv \frac{1}{A} y,
$$

and $z$, because the representative horizontal scale of $\hat{\boldsymbol{u}}$ and $\hat{\theta}$ is expected to be of $\mathrm{O}(A)$.

If we substitute equation (102) into equation (41), we obtain

$$
\hat{w}_{0}=-\hat{\Delta}_{H} \hat{\theta}_{0}
$$

at the leading order of $A$, and

$$
\hat{w}_{1}=-\hat{\Delta}_{H} \hat{\theta}_{1}-\frac{\partial^{2} \hat{\theta}_{0}}{\partial z^{2}}
$$


at the next order of $A$, where

$$
\hat{\Delta}_{H} \equiv \frac{\partial^{2}}{\partial \hat{x}^{2}}+\frac{\partial^{2}}{\partial \hat{y}^{2}}
$$

Substituting equation (102) into equations (39) and (40), we obtain

$$
\begin{gathered}
\frac{\partial}{\partial \hat{x}} \hat{\Delta}_{H} \hat{w}_{0}+\hat{R}_{0} \frac{\partial \hat{\theta}_{0}}{\partial \hat{x}}=0, \\
\frac{\partial}{\partial \hat{y}} \hat{\Delta}_{H} \hat{w}_{0}+\hat{R}_{0} \frac{\partial \hat{\theta}_{0}}{\partial \hat{y}}=0,
\end{gathered}
$$

at the leading order, and

$$
\begin{aligned}
& -\frac{\partial}{\partial z} \hat{\Delta}_{H} \hat{u}_{0}+\frac{\partial}{\partial \hat{x}}\left(\hat{\Delta}_{H} \hat{w}_{1}+\frac{\partial^{2} \hat{w}_{0}}{\partial z^{2}}\right)+\hat{R}_{1} \frac{\partial \hat{\theta}_{0}}{\partial \hat{x}}+\hat{R}_{0} \frac{\partial \hat{\theta}_{1}}{\partial \hat{x}}=0, \\
& -\frac{\partial}{\partial z} \hat{\Delta}_{H} \hat{v}_{0}+\frac{\partial}{\partial \hat{y}}\left(\hat{\Delta}_{H} \hat{w}_{1}+\frac{\partial^{2} \hat{w}_{0}}{\partial z^{2}}\right)+\hat{R}_{1} \frac{\partial \hat{\theta}_{0}}{\partial \hat{y}}+\hat{R}_{0} \frac{\partial \hat{\theta}_{1}}{\partial \hat{y}}=0,
\end{aligned}
$$

at the next order. Substitution of equation (102) into equations (37) and (38) yields

$$
\begin{aligned}
& \frac{\partial \hat{u}_{0}}{\partial \hat{x}}+\frac{\partial \hat{v}_{0}}{\partial \hat{y}}+\frac{\partial \hat{w}_{0}}{\partial z}=0, \\
& \frac{\partial}{\partial \hat{y}} \hat{\Delta}_{H} \hat{u}_{0}-\frac{\partial}{\partial \hat{x}} \hat{\Delta}_{H} \hat{v}_{0}=0,
\end{aligned}
$$

at the leading order.

From equations (103), (105) and (106), we obtain the following equation for $\hat{w}_{0}$ :

$$
\hat{\Delta}_{H}^{2} \hat{w}_{0}=\hat{R}_{0} \hat{w}_{0}
$$

Here, the numerical results shown in figure 13 suggest that for small $A, w$ can be expressed as a product of a function of $\hat{x}$, a function of $\hat{y}$ and a function of $z$ except in the wall layers. Therefore, we assume that $\hat{w}_{0}$ is expressed as

$$
\hat{w}_{0}=\hat{X}_{w}(\hat{x}) \hat{Y}_{w}(\hat{y}) \hat{Z}_{w}(z),
$$

where $\hat{X}_{w}, \hat{Y}_{w}$ and $\hat{Z}_{w}$ are functions of $\hat{x}, \hat{y}$ and $z$, respectively. From the equation obtained by substituting equation (112) into equation (111) and the boundary condition of $\hat{w}_{0}=0$ on the walls, we find that $\hat{X}_{w}$ and $\hat{Y}_{w}$ should satisfy

$$
\begin{array}{ll}
\frac{d^{2} \hat{X}_{w}}{d \hat{x}^{2}}=c_{1} \hat{X}_{w}, & \frac{d^{2} \hat{Y}_{w}}{d \hat{y}^{2}}=c_{2} \hat{Y}_{w}, \\
\hat{X}_{w}=0 \quad \text { at } \quad \hat{x}= \pm \frac{1}{2}, & \hat{Y}_{w}=0 \quad \text { at } \hat{y}= \pm \frac{1}{2},
\end{array}
$$

where $c_{1}$ and $c_{2}$ are constants. Moreover, we obtain the relation

$$
\hat{R}_{0}=\left(c_{1}+c_{2}\right)^{2},
$$


from equations (111)-(113). The even function $\hat{X}_{w}$ and odd function $\hat{Y}_{w}$ that satisfy equation (113) and boundary condition (114) and that yield the smallest $\hat{R}_{0}$ are written as

$$
\hat{X}_{w}=\cos (\pi \hat{x}), \hat{Y}_{w}=\sin (2 \pi \hat{y}),
$$

which give $c_{1}=-\pi^{2}$ and $c_{2}=-4 \pi^{2}$. Therefore, we obtain

$$
\hat{w}_{0}=\cos (\pi \hat{x}) \sin (2 \pi \hat{y}) \hat{Z}_{w}(z), \hat{R}_{0}=25 \pi^{4} .
$$

Here it is noted that although the solution of different symmetry expressed as $\hat{w}_{0}=$ $\cos (\pi \hat{x}) \cos (\pi \hat{y}) \hat{Z}_{w}(z)$ yields $\hat{R}_{0}$ smaller than $25 \pi^{4}$, this solution is not valid because it gives non-zero vertical flux through any horizontal section of the cavity. From equations (103) and (117) and boundary conditions on the walls, we find that $\hat{\theta}_{0}$ is related to $\hat{w}_{0}$ as

$$
\hat{\theta}_{0}=\frac{1}{5 \pi^{2}} \hat{w}_{0} .
$$

The numerical results shown in figure 13 suggest that $u$ and $v$ for small $A$ can be expressed as products of a function of $z$ that is common to $u$ and $v$ and functions of $\hat{x}$ and $\hat{y}$ except in the wall layers. Therefore, we assume that $\hat{u}_{0}$ and $\hat{v}_{0}$ are expressed as

$$
\hat{u}_{0}=P_{u}(\hat{x}, \hat{y}) Z_{h}(z), \hat{v}_{0}=P_{v}(\hat{x}, \hat{y}) Z_{h}(z),
$$

outside the wall layers, where $P_{u}$ and $P_{v}$ are functions of $\hat{x}$ and $\hat{y}$, and $Z_{h}$ is a function of $z$. From equations (109), (110), (117) and (119), we obtain

$$
\begin{aligned}
& Z_{h}=\gamma \frac{d \hat{Z}_{w}}{d z}, \\
& \hat{\Delta}_{H}^{2} P_{u}=-\frac{5 \pi^{3}}{\gamma} \sin (\pi \hat{x}) \sin (2 \pi \hat{y}), \hat{\Delta}_{H}^{2} P_{v}=\frac{10 \pi^{3}}{\gamma} \cos (\pi \hat{x}) \cos (2 \pi \hat{y}),
\end{aligned}
$$

where $\gamma$ is a non-zero constant. If we assume that $P_{u}$ and $P_{v}$ are expressed as products in which their dependences on $\hat{x}$ and $\hat{y}$ are separated, we cannot find their analytic forms that satisfy equation (121) and boundary conditions on the walls. This result suggests that neither $P_{u}$ nor $P_{v}$ can be expressed as such products, and is consistent with the numerical result shown in figure 13.

From equations (103), (104), (107), (108), (117) and (118), we can derive the following equation for $\hat{w}_{1}$ :

$$
\left(\hat{\Delta}_{H}^{2}-\hat{R}_{0}\right) \hat{w}_{1}=15 \pi^{2} \frac{\partial^{2} \hat{w}_{0}}{\partial z^{2}}+\hat{R}_{1} \hat{w}_{0} .
$$

From the solvability condition for inhomogeneous equation (122) having the same homogeneous terms and the same eigenvalue parameter value, the inhomogeneous term of this equation should be 0 . Therefore, by using equation (117), we obtain the following equation:

$$
15 \pi^{2} \frac{d^{2} \hat{Z}_{w}}{d z^{2}}+\hat{R}_{1} \hat{Z}_{w}=0
$$


The even function $\hat{Z}_{w}$ that satisfies equation (123) and the boundary condition of $\hat{Z}_{w}=0$ at $z= \pm 1 / 2$ and also gives the smallest $\hat{R}_{1}$ is written as

$$
\hat{Z}_{w}=\cos (\pi z)
$$

for which

$$
\hat{R}_{1}=15 \pi^{4},
$$

is derived. Therefore, from equations (102), (117) and (125), we obtain

$$
R_{c}=25 \pi^{4} A^{-4}\left(1+\frac{3}{5} A^{2}\right) .
$$

Function $Z_{h}$ is expressed as

$$
Z_{h}=-\gamma \pi \sin (\pi z),
$$

from equations (120) and (124). Therefore, the profile of $Z_{h}$ that is normalized in the same way as the numerical profiles of $z$-dependences of normalized $u$ and $v$ is given by

$$
Z_{h}=\sqrt{2} \sin (\pi z) .
$$

It is noted that this $Z_{h}$ approaches $\sqrt{2}$ as $z$ tends to $1 / 2$ and does not satisfy the boundary condition of $Z_{h}=0$ at $z= \pm 1 / 2$.

The above analytical results agree well with the numerical results for $A \ll 1$ outside the wall layers. That is, in figure 11 we find that the numerical value of $R_{c} A^{4} /\left(25 \pi^{4}\right)$ for small $A$ is well approximated by $1+3 A^{2} / 5$ obtained from equation (126), denoted by a broken line. The numerical profiles of the $z$-dependences of normalized $u$ and $v$ agree well with the analytical profiles given by equation (128), denoted by $\circ$ in figure 13(a), except in the wall layer. The numerical profiles of the $x$-, $y$ - and $z$-dependences of normalized $w$ shown in figures 13(b), (c) and (a) are also well approximated by the analytical profiles of $\cos (\pi x / A), \sin (2 \pi y / A)$ and $\cos (\pi z)$, respectively, denoted by + , except in the wall layer. It is noted that in the wall layers the difference between the numerical and analytical profiles of the $z$-dependences of normalized $u$ and $v$ is large, as found from figure $13(\mathrm{a})$.

\section{Discussion and Conclusions}

Onset of thermal convection of a fluid in a rectangular parallelepiped cavity of small aspect ratios is examined both numerically and analytically under the assumption that its all walls are rigid and of perfect thermal conductance exposed to a vertically linear temperature field. Critical Rayleigh number $R_{c}$ and the steady velocity and temperature fields of most unstable mode are computed by a Galerkin spectral method of high accuracy described in section 3 for aspect ratios $A_{x}$ and $A_{y}$ either or both of which are small.

In section 5.1, we find that if $A_{x}$ is decreased to 0 with $A_{y}$ being kept constant, $R_{c}$ increases proportionally to $A_{x}^{-4}$, the convection rolls of most unstable mode whose axes are parallel to the shorter side walls become narrower, and their number increases 
proportionally to $A_{x}^{-\frac{1}{2}}$. We also observe more frequent changes of the symmetry of most unstable mode for smaller $A_{x}$. However, if $A_{x}=A_{y}=A$ is decreased to 0 , although we again observe the increase in $R_{c}$ proportional to $A^{-4}$, we always obtain the flow pattern of most unstable mode composed of a narrow convection roll, as shown in section 5.2. The existence of the thin wall layers near the top and bottom walls within which rapid variations of horizontal velocity components are found is also observed for small $A_{x}$ or $A$.

Using the dependences of $R_{c}$ and the magnitude and length scale of the velocity field of most unstable mode on $A_{x}$ and $A_{y}$ in the numerical computations, the expressions of $R_{c}$ and velocity fields in the limit of $A_{x} \rightarrow 0$ or $A \rightarrow 0$ are obtained by an asymptotic analysis in section 6 . For example, $R_{c}$ is approximated by $\pi^{4} A_{x}^{-4}$ and $25 \pi^{4} A^{-4}$ in the limits of $A_{x} \rightarrow 0$ and $A \rightarrow 0$, respectively. Moreover, second-order expressions of $R_{c}$ are derived both for $A_{x} \rightarrow 0$ and $A \rightarrow 0$. The analytical expressions of all components of velocity fields for $A_{x} \rightarrow 0$ and the vertical component of velocity fields for $A \rightarrow 0$ are also derived. Moreover, the analytical expressions of boundary lines in the $\left(A_{x}, A_{y}\right)$ plane across which the most unstable mode changes for small $A_{x}$ are derived.

The results of the asymptotic analysis obtained in section 6 agree well with the numerical results for small $A_{x}$ or $A$. That is, the numerical values of $R_{c}$ for small $A_{x}$ or $A$ are well approximated by its analytical expressions. The one-by-one increase in the number of convection rolls of most unstable mode with the decrease in $A_{x}$ for $A_{y}$ being kept constant observed in the numerical computations is perfectly explained by the asymptotic analysis. The numerical boundary lines in the $\left(A_{x}, A_{y}\right)$ plane where the changes of most unstable mode occur for small $A_{x}$ are close to the boundary lines obtained in the asymptotic analysis. The agreement between the numerical velocity field for small $A_{x}$ or $A$ and the analytical expressions of velocity field is quite good except in the wall layers.

In his study under the assumption of finite roll, Davis (1967) pointed out that the flow patterns of most unstable mode are composed of a few narrow convection rolls when one of $A_{x}$ and $A_{y}$ is much smaller than 1, In the present study, similar results are obtained without this assumption by the numerical method of high accuracy, and the details of flow patterns of most unstable mode for such aspect ratios as well as the analytical explanation of them are reported.

One of the characteristic properties of velocity fields when $A_{x}$ or $A$ is small is the rapid variations of horizontal velocity components in the wall layers. The existence of the wall layers is shown clearly in the present numerical study of high accuracy. The asymptotic analysis in the present paper yields the velocity profile that cannot satisfy all the boundary conditions on the top and bottom walls. Therefore, these velocity profiles are not valid in the wall layers. Within the wall layers, a scaling of variables different from the one used in the present study would be necessary. The analytical examination of velocity profiles in the wall layers is left for the future study.

In their studies on the onset of thermal convection in an infinite channel of rectangular cross-section surrounded by rigid walls of perfect thermal conductance, 
Chana and Daniels (1989) numerically obtained the $R_{c}$ and non-dimensionalized critical wavenumber $k_{c}$ that determines the width-to-height ratio of the convection rolls of most unstable mode for several aspect ratio $A_{x}$ of the cross-section. Their results that as $A_{x}$ decreases to $0, R_{c}$ rapidly increases and $k_{c}$ also increases (that is, the convection rolls become narrower) are consistent with the numerical results of the present study for small $A_{x}$ with $A_{y}$ being kept constant. They also performed an asymptotic analysis in the limit of $A_{x} \rightarrow 0$. In this analysis, they adopted the assumptions that are essentially the same as equations (43) and (44), and obtained the asymptotic form of $R_{c}=A_{x}^{-4}\left(\pi^{4}+A_{x} \tilde{R}_{1 \infty}\right)$, which is the same as the result of the present study for $\tilde{y}_{b} \rightarrow 0$. Moreover, the expressions of $\tilde{x}$ - and $z$-dependences of $\tilde{u}_{0}, \tilde{v}_{0}$ and $\tilde{w}_{0}$ shown in section 6.1 of the present study were obtained also in their analysis. Therefore, the result of their analysis is consistent with the analytical result of the present study for $\tilde{y}_{b} \rightarrow \infty$.

The results of the asymptotic analysis for $A_{x} \ll 1$ agree well with the numerical results of $R_{c}$ and the velocity field of most unstable mode outside the wall layers for small $A_{x}$. This agreement suggests that the neglect of the wall layers does not greatly influence the onset of thermal convection for small $A_{x}$ and that the boundary condition on the top and bottom walls is insignificant for such $A_{x}$, which coincides with the assertion of Chana and Daniels (1989) based on their study for both rigid and stress-free top and bottom walls.

It is interesting to examine the effect of imperfection that the cavity is tilted slightly because this effect may cause a remarkable change in the thermal convection of the tall or thin rectangular parallelepiped cavity examined in the present study. The examination of this effect is left for the future study.

\section{References}

Catton, I. 1970. Convection in a closed rectangular region: the onset of motion, ASME J. Heat Transfer, pp.186-188.

Chana, M. S. and Daniels, P. G. 1989. Onset of Rayleigh-Bénard convection in a rigid channel, J. Fluid Mech., 199, pp.257-279.

Daniels, P. G. and Ong, C. F. 1990. Linear stability of convection in a rigid channel uniformly heated from below, Int. J. Heat Mass Transfer, 33, pp.55-60.

Dauby, P. C. and Lebon, G. 1996. Bénard-Marangoni instability in rigid rectangular containers, J. Fluid Mech., 329, pp.25-64.

Davies-Jones, R. P. 1970. Thermal convection in an infinite channel with no-slip sidewalls, J. Fluid Mech., 44, pp.695-704.

Davis, S. H. 1967. Convection in a box: linear theory, J. Fluid Mech., 30, pp.465478. 
Edwards, B. F. 1988. Crossed rolls at onset of convection in a rigid box, J. Fluid Mech., 191, pp.583-597.

Fukazawa, Y. and Funakoshi, M. 2015. Onset of thermal convection and its flow patterns in a rectangular cavity, Fluid Dyn. Res., 47, 065505.

Gelfgat, A. Y. 1999. Different modes of Rayleigh-Bénard instability in two- and three-dimensional rectangular enclosures, J. Comp. Phys., 156, pp.300-324.

Gershuni, G. Z. and Zhukhovitskii, E. M. 1972. "Convective Stability of Incompressible Fluids," (Nauka, Moscow, English Translation: Keter Publishing House, Jerusalem, 1976) Chapter IV.

Kirchartz, K. R. and Oertel Jr, H. 1988. Three-dimensional thermal cellular convection in rectangular boxes, J. Fluid Mech., 192, pp.249-286.

Koschmieder, E. L. 1966. On convection on a uniformly heated plane, Beitr. Phys. Atmos., 39, pp.1-11.

Koschmieder, E. L. 1993. "Bénard Cells and Taylor Vortices," (Cambridge University Press) Section 5.2.

Lappa, M. 2010. "Thermal Convection: Patterns, Evolution and Stability," (John Wiley \& Sons) Chapter 4.

Mizushima, J. and Matsuda, O. 1996. (in Japanese) Res. Inst. Appl. Math., Kyoto Univ. Kokyuroku, 970, pp.1-9.

Mizushima, J. and Matsuda, O. 1997. Onset of 3D thermal convection in a cubic cavity, J. Phys. Soc. Jpn., 66, pp.2337-2341.

Mizushima, J. and Nakamura, T. 2003. Onset of three-dimensional thermal convection in a rectangular parallelepiped cavity, J. Phys. Soc. Jpn., 72, pp.197200 .

Puigjaner, D., Herrero, J., Giralt, F. and Simó, C. 2006, Bifurcation analysis of multiple steady flow patterns for Rayleigh-Bénard convection in a cubical cavity at $\operatorname{Pr}=130$, Phys. Rev. E, 73, 046304.

Puigjaner, D., Herrero, J., Simó, C. and Giralt, F. 2008. Bifurcation analysis of steady Rayleigh-Bénard convection in a cubical cavity with conducting sidewalls, J. Fluid Mech., 598, pp.393-427.

Sherman, M. and Ostrach, S. 1966. On the principle of exchange of stabilities for the magnetohydrodynamic thermal stability problem in completely confined fluids, J. Fluid Mech., 24, pp.661-671.

Stork, K. and Müller, U. 1972. Convection in boxes: experiments, J. Fluid Mech., 54, pp.599-611. 